\title{
Erbbaurecht als Anlageform für institutionelle Investoren
}

\author{
Steffen Sebastian · Dominik Wagner · Carsten Fritz
}

Eingegangen: 16. Juli 2019 / Überarbeitet: 17. Januar 2020 / Angenommen: 5. Februar 2020 / Online publiziert: 10. März 2020

(C) Der/die Autor(en) 2020

Zusammenfassung Das Erbbaurecht ist eine probate Alternative zum klassischen Volleigentum. Vorrangig von Kommunen und Stiftungen angewandt, ist diese Form der Grundstücksteilung in vielen entwickelten Ländern die typische Form des Immobilienerwerbs. Vor dem Hintergrund des niedrigen Zinsumfelds suchen institutionelle Investoren nach alternativen Anlagevehikeln mit zufriedenstellenden RenditeRisiko-Verhältnissen. Diese Arbeit untersucht die Marktakzeptanz des deutschen Erbbaurechts sowie mögliche Einflüsse aus Großbritannien als einem der höchstentwickelten Märkte für Erbbaurecht. Unsere Analyse zeigt, dass das Erbbaurecht ein sinnvolles Nischenprodukt für institutionelle Investoren zur Generierung von Mehrwerten darstellt. Der individuelle Nutzen für Investoren mit deutschem Investmentfokus hängt jedoch stark von der konkreten Vertragsgestaltung ab. Der britische Markt stellt für deutsche Immobilienfonds aktuell aufgrund der höheren Liquidität und Transparenz des Marktes eine geeignetere Anlageallokation dar.

Schlüsselwörter Erbbaurecht · Institutionelle Investoren · Erbbauzins · Volleigentum · Überrenditen 


\title{
Leasehold as an asset class for institutional investors
}

\begin{abstract}
Leasehold is a proven alternative to a common investment in the form of full ownership. Primarily applied by municipalities and foundations, this form of bifurcation is the typical form of real estate acquisition in many developed countries. Given the low interest rate environment, institutional investors are looking for alternative investment vehicles with satisfactory risk to return ratios. At first, this paper examines the market acceptance of German leaseholds (called Erbbaurecht) and possible influences from the UK as one of the most developed markets of leasehold rights. Our analysis shows that leasehold law is a suitable niche product for institutional investors to generate excess returns even in times of low interest rates. The individual benefit for investors with a German investment focus, however, strongly depends on the practical contract design. In comparison, the UK market currently offers a more appropriate asset allocation for German real estate funds due to the higher liquidity and transparency of the market.
\end{abstract}

Keywords Leasehold · Ground lease - Ground rent · Asset class · Excess returns

\section{Erbbaurecht für institutionelle Investoren}

Das anhaltend niedrige Zinsumfeld zwingt institutionelle Investoren dazu, nach alternativen Anlagen zu suchen, die sowohl den Renditeanforderungen genügen als auch mit einem konservativen Risikoprofil vereinbar sind. Versicherungsgesellschaften oder Pensionskassen haben naturgemäß langfristige Anlagehorizonte, da diese ebenso langfristigen Verbindlichkeiten gegenüberstehen. Zusätzlich sehen Regulationen vor, dass ein Großteil des zur Verfügung stehenden Kapitals lediglich in Assets mit möglichst sicherem Cashflow investiert werden darf. Auskömmliche Renditen werden derzeit nur in riskanten Anlageklassen oder mit Immobilieninvestments realisiert. Geeignete Anlagemöglichkeiten werden hier jedoch durch die anhaltend hohe Nachfrage knapper. Naturgemäß geraten daher immer wieder Nischenprodukte wie Mikrowohnen, Parkhäuser oder Gesundheitsimmobilien in den Fokus der Investoren. Ein weiteres Nischenprodukt könnten Erbbaurechte und Erbbaurechtsgrundstücke im klassischen Investitionssegment der institutionellen Anleger sein.

Das Erbbaurecht bringt rechtliche und ökonomische Besonderheiten und Einschränkungen bei Erwerb, Finanzierung, Verwaltung und insbesondere bei der Veräußerung mit sich. Nicht zuletzt sorgt die zusätzliche Komplexität des Konzeptes Erbbaurecht dafür, dass sowohl Erbbaurechte als auch Erbbaurechtsgrundstücke bei institutionellen Investoren grundsätzlich als Investition ausgeschlossen werden. Allerdings stellt gerade diese Komplexität des Produktes eine Chance für spezialisierte Marktteilnehmer dar. Institutionelle Investoren, die diese spezifischen Kenntnisse generieren können und über passende Investmenthorizonte verfügen, haben am Markt potenziell komparative Vorteile. Für institutionelle Anleger ist außerdem neben der Position des Erbbaurechtsgebers grundsätzlich auch die Position des Erbbaurechtsnehmers und Vermieters denkbar. Zusätzlich lässt sich durch die Aufspaltung eines Grundstücks in die Einzelkomponenten, d.h. des Erbbaurechts und des Erbbau- 
rechtsgrundstücks, potenziell ein Wertgewinn erzielen, da die Trennung zwei neue Investitionsgüter mit unterschiedlichem Risiko-Rendite-Profil generiert und so eine effizientere Allokation unter den Marktteilnehmern ermöglicht. Hieraus könnten sich neue Geschäftsfelder für die Anbieter von spezialisierten Investitionsprodukten ergeben.

In Deutschland werden Erbbaurechte primär von Stiftungen sowie kirchlichen oder kommunalen Institutionen vergeben. In jüngster Zeit wird verstärkt über die Vergabe von Erbbaurechten durch die Kommunen als wohnungspolitische Maßnahme, vor allem in Großstädten, diskutiert, ${ }^{1}$ wodurch vermehrt Erbbaurechte auf den Markt kommen könnten. Erbbaurechtsnehmer sind üblicherweise Privatpersonen, wobei auch große gewerbliche Erbbaurechte (bspw. Hafenanlagen, Flughäfen, Fußballstadien etc.) zu beobachten sind. Das Marktvolumen von Erbbaurechten hat hierbei insbesondere in Großstädten aufgrund der Preissteigerungen des Baulands zugenommen. Institutionelle Anleger sind derzeit am Markt weder als Erbbaurechtsgeber noch als Erbbaurechtsnehmer in signifikantem Umfang präsent.

Im Gegensatz zu Deutschland ist in Großbritannien das Konzept des Erbbaurechts (Leasehold) weitverbreitet. Vor allem Leasehold an Wohnungen erfreut sich in Großbritannien steigender Beliebtheit. Landesweit unterliegt fast jede fünfte Immobilie dem Leasehold. Zudem ist der Markt, u.a. durch staatliche Marktstudien, deutlich transparenter.

Diese Arbeit macht sich zum Ziel, mit Hilfe von deskriptiven Analysen Informationslücken bezüglich des deutschen Erbbaurechtsmarktes zu schließen und den Nutzen des Produktes Erbbaurecht für institutionelle Investoren anschaulich darzustellen. Im Folgenden werden in Abschn. 2 zunächst die Grundzüge des Erbbaurechts dargestellt, bevor in Abschn. 3 die verfügbaren Marktinformationen zum Erbbaurecht in Deutschland aufgeführt und mit Großbritannien als Referenz verglichen werden. In Abschn. 4 wird auf direkte und indirekte Investitionsformen, insbesondere auf spezialisierte Fonds, eingegangen. Um das Potenzial eines Mehrwertes durch Aufspaltung zu überprüfen, werden in Abschn. 5 die etablierten Verfahren zur Wertermittlung in Deutschland den standardisierten Verfahren in Großbritannien gegenübergestellt. Abschließend soll in Abschn. 6 diskutiert werden, ob es sich beim Erbbaurecht trotz der Marktenge in Deutschland derzeit um ein sinnvolles Investitionsobjekt handelt.

\section{Grundlagen des Erbbaurechts}

Das Konzept des Erbbaurechts existiert bereits seit Jahrhunderten in Europa, etwa in Frankreich, den Niederlanden, Österreich oder der Schweiz. Vor allem in Großbritannien ist es weitverbreitet. Rechtlich kodifiziert ist es in Deutschland seit $1900^{2}$.

\footnotetext{
${ }^{1}$ So wollen bspw. München und Hamburg vorrangig vermehrt Grundstücke mit Erbbaurecht vergeben, vgl. SPD Bürgerschaftsfraktion Hamburg (2019); Runder Tisch Liegenschaftspolitik (2017, S. 14-15); Landeshauptstadt München: Referat für Stadtplanung und Bauordnung (2017).

2 Vgl. v. Oefele et al. (2016), Rn. 2 f.
} 


\subsection{Deutschland}

Nach deutschem Recht ist ein Erbbaurecht nach der Legaldefinition des $\$ 1$ Abs. 1 ErbbauRG die Belastung eines Grundstücks durch das Recht, ,auf oder unter der Oberfläche eines Grundstücks ein Bauwerk zu haben“. ${ }^{3}$ Als grundstücksgleiches Recht ist das Erbbaurecht frei handelbar, übertragbar, vererblich und beleihbar ${ }^{4}$. Die Bestellung eines Erbbaurechts ermöglicht demzufolge die rechtliche Trennung von Grundstücks- und Gebäudeeigentum. ${ }^{5}$ Dies ermöglicht entsprechend die getrennte Vermarktung dieser beiden Rechte. Das Bauwerk wird dabei wesentlicher Bestandteil des Erbbaurechts, unabhängig davon, ob es schon besteht oder noch gebaut wird. ${ }^{6}$ Erbbauberechtigte können einzelne, aber auch mehrere natürliche oder juristische Personen sein. ${ }^{7}$

Das Erbbaurecht wird typischerweise über sehr lange Zeiträume vergeben. Bei wohnwirtschaftlicher Nutzung sind es üblicherweise 60 bis 99 Jahre, bei gewerblicher Nutzung 40 bis 50 Jahre. Grundsätzlich sind auch längere Fristen für gewerbliche Erbbaurechte denkbar. Während der Laufzeit des Erbbaurechts erhält der Erbbaurechtsgeber vom Erbbaurechtsnehmer den sogenannten Erbbauzins, der ins Erbbaugrundbuch eingetragen wird. In der Praxis ist die dingliche Vereinbarung als wiederkehrende Leistungen gemäß $\S 9$ Abs. 1 ErbbauRG üblich. ${ }^{8}$ Der Erbbauzins ist nicht Inhalt des Erbbaurechts, sondern nur eine Belastung desselbigem. ${ }^{9}$ Ein solcher Erbbauzins ist Bestandteil des Grundstücks, wodurch zukünftige Erbbauzinszahlungen nicht gehandelt oder verpfändet werden können. ${ }^{10}$ Der anfängliche Erbbauzins ist in seiner Höhe unbeschränkt und kann frei zwischen den Vertragsparteien vereinbart werden. In der Regel wird ein bestimmter Prozentsatz des Verkehrswerts des belasteten Grundstücks als Erbbauzins vereinbart ${ }^{11}$ und liegt bei privat genutzten Erbbaurechten meist bei 3 bis $5 \%$, bei gewerblich genutzten Erbbaurechten normalerweise etwas höher.

Ein komplexer Bereich des Erbbaurechts ist die Frage, ob und in welchem Umfang eine Wertsicherung des vereinbarten Erbbauzinses während der Laufzeit möglich ist. ${ }^{12}$ Nach neuer Rechtslage genügt die eindeutige Bestimmbarkeit des Erb-

\footnotetext{
${ }^{3}$ Erbbaurecht kann in acht Bestellungen gegliedert werden: Erbbaurecht, Gesamterbbaurecht, Eingeschränktes Erbbaurecht, Untererbbaurecht, Nachbarerbbaurecht, Wohnungserbbaurecht, Teilerbbaurecht, Eigentümererbbaurecht. Vgl. \$39 SachenRBerG \& §30 WEG \& BGHZ 62, 179 in NJW 1974, 37.

4 Nähere Informationen zur Beleihung sind im Anhang aufgeführt.

5 Vgl. Böttcher (2017, S. 1).

6 Vgl. §12 Abs. 1 ErbbauRG.

7 Vgl. Grizwotz (2017), §1 Rn. 15 f.

8 Rechtlich zulässig sind auch Einmalzahlungen oder eine Umsatzbeteiligung an einem Gewerbebetrieb auf dem Grundstück, vgl. BGH, Urteil v. 27.02.1970, V ZR 49/67.

9 Vgl. Hustedt und Ingenstau (2014), §9 Rn. 10.

10 Vgl. Böttcher (2017, S. 87).

11 Vgl. v. Oefele et al. (2016), §6 Rn. $65 \mathrm{f}$.

12 Vgl. Schmenger (2006, S. 75). Bei bis zum 30.09.1994 eingetragenen Erbbaurechten konnte nach dem damaligen $\$ 9$ Abs. 2 ErbbauRG a.F. der Erbbauzins für die gesamte Dauer des Erbbaurechts im Voraus bestimmt werden, d.h. es wäre eine grundbuchmäßig geschützte Wertsicherung möglich gewesen. Üblich war jedoch die rein schuldrechtliche Vereinbarung einer Wertsicherung in Verbindung mit dem Eintrag
} 
bauzinses. Strengere Regelungen greifen bei Erbbaurechten zu Wohnzwecken. Die Voraussetzungen und der Bewertungsmaßstab für die Veränderung des Erbbauzinses unterliegen der Vertragsfreiheit. ${ }^{13}$ Üblich sind index- beziehungsweise leistungsbezogene Klauseln, d.h. die Anpassung kann an den Verbraucherpreisindex oder im gewerblichen Fall auch an die Wertsteigerung des Bodenwerts gekoppelt sein. Alternativ besteht auch die Möglichkeit einer Neuverhandlungsklausel, die es einer Vertragspartei erlaubt, die Neuverhandlung der Höhe des Erbbauzinses einzufordern, sofern im Voraus definierte Voraussetzungen erfüllt werden. Der §9a ErbbauRG schränkt diese Anpassungsmöglichkeiten für Erbbaurechte, die Wohnzwecken dienen, allerdings ein. ${ }^{14}$ So dürfen Erhöhungen nur nach jeweils drei Jahren durchgeführt werden und beziehen die Entwicklung der Inflationsrate mit ein. Bei gewerblichen Bauten ist die Anpassung jährlich möglich. Die Erhöhung des Erbbauzinses unterliegt dabei keiner prinzipiellen Beschränkung.

Die Ermittlung des Beleihungswertes des Erbbaurechtes selbst erfolgt in der Praxis meist durch inländische Verfahren, bspw. das Münchner Verfahren. Eine ausführliche Darstellung findet sich im Anhang.

Mit Ablauf der vereinbarten Laufzeit des Erbbaurechtsvertrages wird das auf dem Grundstück errichtete Gebäude zum Eigentum des Grundstückeigentümers. Der Erbbaurechtsnehmer kann eine Entschädigung in vorher vereinbarter Höhe erhalten. Im privaten Bereich sind Entschädigungshöhen von 2/3 bis $100 \%$ des Verkehrswertes des Gebäudes der Regelfall, wobei es im sozialen Wohnungsbau eine Sonderregelung gibt, nach welcher die Entschädigung bei mindestens 2/3 des allgemeinen Wertes der Bebauung liegt. ${ }^{15}$ Besteht keine vertragliche Vereinbarung, müssen sich die Parteien gegebenenfalls durch ein Rechtsverfahren über die Höhe einigen. Die Entschädigungshöhe orientiert sich an dem Verkehrswert, der nach ImmoWertV ermittelt wird. ${ }^{16}$ Es ist möglich, diese Zahlung zu umgehen, indem der Erbbaurechtsgeber dem Nutzer vor Zeitablauf anbietet, das Erbbaurecht zu verlängern. „Schlägt der Nutzer dieses Angebot aus, erlischt der Anspruch auf Entschädigung. “17,18 Sonderregelungen treten beim sogenannten Heimfall ein. Sollte der Gebäudeeigentümer mit der Erbbauzinszahlung in Höhe mindestens zweier Jahresbeiträge in Verzug sein

einer Vormerkung, vgl. Böttcher (2017, S. 87-89). Für die Anpassung ist neben der ersten Rangstelle des Erbbauzinses eine Vormerkung notwendig, nach der auch der neue angepasste Erbbauzins dort eingetragen werden kann. Dafür ist jeweils ein notariell beglaubigter Nachtragsvertrag erforderlich, der den neuen Erbbauzins ausweist und der beim Grundbuchamt eingereicht wird. Der neue Erbbauzins wird dinglich im Grundbuch eingetragen. Dies ist bis heute bei allen vor 01.10.1994 abgeschlossenen Erbbaurechten notwendig, soweit diese Verträge nicht auf die neue Rechtslage umgestellt werden konnten. Diese Umstellung ist allerdings nicht ohne Weiteres möglich, da dafür die Zustimmung der nachrangigen Gläubiger benötigt wird (vgl. DNot-Report 2001, S. 177,179).

13 Vgl. v. Oefele et al. (2016), §6 Rn. 96.

14 Hier zeigen sich Parallelen zum Mieterschutz bei der Wohnraumvermietung.

15 Vgl. Meusel (2017, S. 27).

16 Vgl. §§15-23 ImmoWertV.

17 Vgl. Hüren (2015).

18 Der Grundstückseigentümer kann finanzielle Lasten, die er eventuell nicht tragen kann, abwenden, indem er das Erbbaurecht auf die Standdauer des Bauwerkes verlängert, dann allerdings ohne jegliche Anpassungsmöglichkeit eines ggf. sehr niedrigen Erbbauzinses. Insofern ist dies in der Praxis meist eine eher unattraktive Option des Grundstückseigentümers. 
oder seine Insolvenz in Kraft treten, so kann der Heimfall durch den Erbbaurechtsgeber betrieben werden. Sofern der Erbbauzins erstrangig gesichert ist und daneben nachrangige Darlehen bestehen, kann es für den Grundstückseigentümer vorteilhaft sein, Zwangsvollstreckungsmaßnahmen zu betreiben. Die nachrangigen Darlehen können dann ganz oder teilweise wegfallen. Beim Heimfall bleiben diese hingegen immer bestehen. ${ }^{19}$

Beim Heimfall kann der Erbbaurechtsgeber das Gebäude verwerten, um seine Ansprüche zu decken, und als Entschädigung den Verkehrswert des Gebäudes mit einem Abschlag zahlen. Die konkrete Ausgestaltung richtet sich nach dem jeweiligen Erbbaurechtsvertrag.

\subsection{Großbritannien}

In Großbritannien hat das Erbbaurecht (Leasehold beziehungsweise Ground Lease) hohe Akzeptanz. Aufgrund von Fehlentwicklungen in der jüngsten Vergangenheit wurde eine Reihe von Reformen durchgeführt.

Der Ursprung des britischen Grundstücksrechts liegt im Feudalwesen des 11. Jahrhunderts. ${ }^{20}$ Infolgedessen ist das gesamte Grundvermögen in England und Wales formal Eigentum der Krone. ${ }^{21}$ Der Erwerb von Boden ist nur in Form von Besitzrechten möglich. Das umfassendste Besitzrecht ist das Freehold, das sich jedoch de facto nicht vom Volleigentum nach deutschem Recht unterschiedet. ${ }^{22}$ Ein Leasehold ist demgegenüber ein zeitlich begrenztes Besitzrecht mit sehr langen Laufzeiten von bis zu 199 Jahren. Mit kurzen Laufzeiten von ggf. wenigen Jahren oder nur Monaten kann ein Leasehold auch Mietverträgen nach deutschem Recht ähneln. ${ }^{23}$ Darüber hinaus ist auch das Einräumen von Unter-Leases möglich.

Dem deutschen Erbbaurecht entsprechen Long-Leases mit Laufzeiten von mehreren Jahrzehnten. Im Unterschied zum deutschen Erbbaurecht ist bei solchen langlaufenden Leaseholds für Wohnimmobilien eine anfängliche Einmalzahlung (Premium) zu leisten, die nahezu dem Preis für das Freehold-Eigentum entspricht. ${ }^{24}$ Darüber hinaus wird üblicherweise ein jährliches Entgelt gezahlt, die sogenannte Ground Rent. ${ }^{25}$ Am Ende der Laufzeit fällt das Grundstück samt Gebäude entschädigungslos an den Freehold-Eigentümer zurück. ${ }^{26}$ Instandhaltungen sind vom Landlord durchzuführen, die Kosten hierfür trägt jedoch der Inhaber des Leasehold.

\footnotetext{
$19 \S \S 32,33$ ErbbauRG.

20 Vgl. Dixon (2016).

21 Die Ausführungen beziehen sich auf den englischen und walisischen Landesteil des Vereinigten Königreichs. In Schottland wurden Erbbaurechte per Gesetz im Jahr 2015 zu Volleigentum umgewandelt. In Nordirland herrscht historisch bedingt eine andere Gesetzgebung, vgl. Collinson (2017).

22 Vgl. Dixon (2016, S. 8) oder Eisenhauer (1997).

23 Als drittes Besitzrecht existiert seit dem Leasehold Reform Act 2002 das Commonhold, welches vergleichbar mit dem deutschen Wohnungs- oder Teileigentum ist. Dieses ist jedoch bisher praktisch bedeutungslos, da es in über einem Jahrzehnt nur in 161 Fällen angewendet wurde, vgl. Waldner und Kopp (2015) oder Driscoll (2016).

24 Vgl. Waldner und Kopp (2015), Rn. 50.

25 Vgl. Dixon (2016, S. 228f).

26 Vgl. Waldner und Kopp (2015), Rn. 50 oder Eisenhauer (1997, S. 25).
} 
Solche langfristigen Leaseholds sind vor allem bei Wohnimmobilien in großen Städten verbreitet.

Allerdings ist der Leaseholder keineswegs schutzlos, da er das Recht auf das sogenannte Enfranchisement hat. Das Enfranchisement entstammt einer der Sonderregelungen im englischen Erbbaurecht, welches mit dem Leasehold Reform Act 1993 in Kraft getreten ist. Dabei ist es möglich - selbst entgegen dem Willen des Eigentümers -, das Grundstück zum gegenwärtigen Wert des Leaseholds und belasteten Freeholds plus dem hälftigen Wert des sogenannten Marriage Value zu erwerben (vgl. Anhang, Kapitel 9.5 Marriage Value, S. 21). Dieses Recht ist jedoch an Bedingungen geknüpft. So können nur Privatpersonen die Möglichkeit wahrnehmen und müssen den Wohnraum dafür auch zwei Jahre selbst genutzt haben. Dies führt dazu, dass Freeholder dazu tendieren, Leaseholds nur für einzelne Wohnungen zu vergeben. Das Enfranchisement ist in diesem Fall weniger wahrscheinlich, da eine Übernahme dann nur von den Leaseholdern aller Wohnungen in einem Gebäude gleichzeitig möglich ist (Collective Enfranchisement). ${ }^{27} \mathrm{Im}$ Rahmen einer individuellen Vertragsgestaltung ist es zudem möglich, Anreize für ein Enfranchisement zu bestimmten Zeiten zu setzen, die für den Freeholder vorteilhaft sind. So kann zumindest die Wahrscheinlichkeit einer Übernahme gesteuert werden. ${ }^{28}$

Es ist beabsichtigt, die gesetzlichen Regelungen für Leasehold zu ändern. So schlug die britische Regierung im Juli 2017 vor, den Leasehold für Neubauten und Erschließungen komplett abzuschaffen. Lediglich Bestandswohnungen sollten noch unter dem Leasehold vergeben werden können. Allerdings soll die Ground Rent auf ein Minimum, bestenfalls nahe null, reduziert werden (peppercorn rent). Aktuell gibt es Vorschläge bezüglich eines Leasehold-Verbots für fast alle Neubauten. Zusätzlich wird diskutiert, die Ground Rent für Langzeit-Leaseholds auf null zu setzen. Grund dafür sind vor allem spekulative Investoren, die stark zum rapiden Wachstum der Ground Rent beitragen. Aufgrund des grundsätzlich liberalen Rechts des britischen Wohnungsmarkts ist es beispielsweise möglich, Klauseln in den Leasehold einzubauen, die für eine Verdopplung der Ground Rent in regelmäßigen Abständen sorgen. Dies führt dazu, dass Erbbaurechte mit einer solchen Leasehold-Klausel unverkäuflich werden. Weitere Reformen waren für Sommer 2018 angekündigt. ${ }^{29}$ Im Oktober 2018 erschien ein neues Konsultationspapier der Regierung, welches erneut potenzielle Änderungen des Gesetzes vorschlägt, die nach eigenen Angaben frühestens Mitte 2020 in Kraft treten werden. Seit Dezember 2018 läuft im Auftrag des Ministeriums für Wohnungswesen parallel ein Projekt des Cambridge Centre of Housing \& Planning Research zur Datenerhebung und Validierung der Kostenhöhe von Leaseholds sowie deren Schwankungen. Am 12. April 2019 veröffentlichte das britische Unterhaus ein Briefing Paper für die Reform des Leaseholds, welche die „möglichen Reformen“ vorstellt. Somit kann festgehalten werden, dass selbst

\footnotetext{
27 Vgl. The Leasehold Reform Regulations (1993).

28 Eine Regelung für den zwangsweisen Verkauf an den jeweiligen Erbbauberechtigen sieht das deutsche Gesetz im $\$ 2$ Nr. 7 ErbbauRG vor. Diese Regelung tatsächlich auch vertraglich zu vereinbaren, wird sich in Deutschland aber als sehr schwierig erweisen.

29 Für eine ausführliche Darstellung der Historie zum Verlauf der Leasehold Reform siehe Department for Communities and Local Government (2017b).
} 
für den britischen Markt erst eine valide Datenbasis geschaffen werden muss und viele parlamentarische Einrichtungen Vorschläge diskutieren, die selbst bei einer Verabschiedung der Gesetzesnovellen in 2020 erst deutlich später ihre tatsächliche Wirkung entfalten werden. ${ }^{30}$

\section{Marktdaten}

\subsection{Deutschland}

Marktvolumen Das Marktvolumen in Deutschland ist unbekannt, verlässliche Schätzungen oder repräsentative Informationen zum Erbbaurecht in Deutschland sind nicht vorhanden; auch eine Anfrage beim Arbeitskreis der Gutachterausschüsse und Oberen Gutachterausschüsse in Deutschland (AK-OGA) ergab, dass für Deutschland keine Angaben verfügbar sind. Gemäß einer Studie der Kölner Initiative Erbbaurecht aus dem Jahre 2009 stehen rund 5\% der Wohnbaufläche in Deutschland auf Erbbaurechtsgrundstücken. ${ }^{31}$ Gemäß von Oefele und Winkler (2012) sind circa 3\% der für Wohnzwecke genutzten Grundstücke in Erbbaurecht vergeben. ${ }^{32}$ Vergleichbare Angaben zu Gewerbeflächen fehlen. Aufgrund des Mangels von Statistiken zur Thematik des Erbbaurechts in Deutschland führte der deutsche Erbbaurechtsverband (2018) eine Umfrage unter ca. 600 Institutionen durch. ${ }^{33}$ Daraus ergab sich eine Stichprobe von 87 Befragten, darunter vorherrschend Kommunen. Von diesen 87 Teilnehmern der Studie werden gesamt 90.038 Erbbaurechte gehalten. Der Großteil der Erbbaurechtsgeber verwaltet jeweils weniger als 500 Erbbaurechte. Die gehaltenen Erbbaurechte sind vorwiegend Erbbaurechte für Wohnzwecke (69\%). Kirchliche Einrichtungen, welche einen großen Teil der deutschen Erbbaurechtsgeber darstellen, waren nur zu einem geringen Teil vertreten.

Jones Lang LaSalle veröffentlichte im März 2019 eine Studie zu Erbbaurechten in den deutschen Top-7 Städten. Darin wird der Investmentmarkt anhand der Transaktionsvolumen für den Zeitraum 2016-2018 erfasst. In Summe werden 2400 gewerbliche Immobilienkäufe beobachtet, wobei 47 Transkationen mit Erbbaurecht identifiziert werden. Gemessen an der Anzahl entspricht dies 2\% der Transaktionen, bzw. 4,7\% des Werts aller Transaktionen. ${ }^{34}$

Weder die Daten des deutschen Erbbaurechtsverbandes noch die von JLL erlauben Hochrechnungen, um das Volumen des gesamten deutschen Marktes zu approximieren. Auch existieren unseres Wissens nach keine Informationen über das Volumen von Direktinvestitionen anderer deutscher institutioneller Anleger. ${ }^{35}$ Bei der Auswer-

\footnotetext{
30 Vgl. Wilson und Barton (2019).

31 Vgl. Licher (2009).

32 Vgl. von Oefele und Winkler (2012, S. 19).

33 Deutscher Erbbaurechtsverband e. V. (2018).

34 Vgl. Jones Lang LaSalle (2019, S. 18).

35 Entsprechende Anfragen bei BaFin und BVI blieben bislang unbeantwortet. Der GdV teilte mit, dass keine Unterscheidung zwischen Erbbaurecht und Volleigentum erfolgt.
} 
Tab. 1 Anteil Erbbaurechte Publikums-AIF

\begin{tabular}{|c|c|c|c|c|c|}
\hline Fonds & $\begin{array}{l}\text { Datum } \\
\text { Bericht }\end{array}$ & $\begin{array}{l}\text { Anzahl } \\
\text { Erbbau- } \\
\text { rechte }\end{array}$ & $\begin{array}{l}\text { Anteil an der } \\
\text { gesamten } \\
\text { Grundstücks- } \\
\text { fläche }(\%)\end{array}$ & $\begin{array}{l}\text { Anteil am } \\
\text { Immobili- } \\
\text { envermögen } \\
(\%)\end{array}$ & $\begin{array}{l}\text { Erbbaurechtsnehmer } \\
\text { oder -geber }\end{array}$ \\
\hline HausInvest & 31.03 .2018 & 17 & $31,87^{\mathrm{a}}$ & 0,03 & Erbbaurechtsnehmer \\
\hline $\begin{array}{l}\text { Grundbesitz } \\
\text { europa RC }\end{array}$ & 30.09 .2017 & 16 & 15,32 & 27,15 & Erbbaurechtsnehmer \\
\hline $\begin{array}{l}\text { UniImmo: } \\
\text { Deutschland }\end{array}$ & 31.03 .2018 & 6 & 0,94 & 1,87 & Erbbaurechtsnehmer \\
\hline $\begin{array}{l}\text { UniInstitutional } \\
\text { European } \\
\text { Real Estate }\end{array}$ & 31.03 .2018 & 6 & 2,52 & 11,62 & Erbbaurechtsnehmer \\
\hline $\begin{array}{l}\text { Grundbesitz } \\
\text { global RC }\end{array}$ & 30.09 .2017 & 5 & 29,71 & 14,31 & $\begin{array}{l}\text { Erbbaurechtsnehmer } \\
\text { (teilweise gleichzeitig } \\
\text { Erbbaurechtsnehmer } \\
\text { und -geber) }\end{array}$ \\
\hline $\begin{array}{l}\text { UniImmo: } \\
\text { Global }\end{array}$ & 31.03 .2018 & 4 & 21,49 & 4,84 & Erbbaurechtsnehmer \\
\hline $\begin{array}{l}\text { UniImmo: } \\
\text { Europa }\end{array}$ & 31.03 .2018 & 3 & 16,14 & 2,11 & Erbbaurechtsnehmer \\
\hline $\begin{array}{l}\text { Aachener } \\
\text { Spar- und } \\
\text { Stiftungs- } \\
\text { Fonds }\end{array}$ & 31.03.2018 & 2 & 15,70 & 11,6 & Erbbaurechtsnehmer \\
\hline $\begin{array}{l}\text { UBS (D) } \\
\text { Euroinvest } \\
\text { Immobilien }\end{array}$ & 31.08 .2017 & 2 & 4,08 & 12,45 & $\begin{array}{l}\text { Erbbaurechtsnehmer } \\
\text { und -geber }\end{array}$ \\
\hline $\begin{array}{l}\text { Aachener } \\
\text { Grund-Fonds } \\
\text { Nr. } 1\end{array}$ & 30.09 .2013 & 1 & 0,78 & 1,22 & Erbbaurechtsnehmer \\
\hline
\end{tabular}

aDie Grundstücke in Erbbaurecht sind teilweise sehr groß, woraus sich vermutlich der deutliche Unterschied zum wertmäßigen Anteil ergibt. Beispielsweise wird für das Objekt Westfield London (UK) eine Fläche von $184.935 \mathrm{~m}^{2}$ ausgewiesen. Ein weiteres Grundstück in Izmir (Türkei) mit einer Fläche von $200.000 \mathrm{~m}^{2}$ wird als Teilerbbaurecht ausgewiesen

${ }^{\text {b}}$ Für das Objekt Chaussée de la Hulpe sowohl Erbbaurechtsnehmer als auch -geber. Hierzu wird nur die Nutzfläche, nicht aber die Grundstücksfläche ausgewiesen. Für das Objekt FAC1 liegt ein Teilerbbaurecht i. H. v. $40 \%$ der Grundstücksfläche vor. Der Portfolioanteil wird prozentual approximiert

tung aktueller Geschäftsberichte von 18 Publikumsfonds konnten wir bei 10 Fonds Investitionen in Erbbaurechtsgrundstücke identifizieren.

Der Anteil von Erbbaurechten am gesamten Immobilienvermögen ist je nach Fonds sehr unterschiedlich und beträgt zwischen 0,03 und 27,15\%. Dabei nehmen sie bis auf die beiden oben angegebenen Ausnahmen stets die Position des Erbbaurechtsnehmers ein.

Marktentwicklung Aufgrund des wenig entwickelten Marktes gibt es in Deutschland nur einige kleinere Anbieter von Dienstleistungen zu Erbbaurechten. Dazu zählen beispielsweise die Immofori AG, Continuum Capital Investment Management GmbH, GLC Ground Lease Concepts GmbH, Operatio Vermögensverwaltung, DGR Grundstücksverwaltung sowie die Liemak IT GmbH, welche sich zusätzlich mit der 
Tab. 2 Übersicht Entwicklung Erbbauzinssätze in Deutschland

\begin{tabular}{lll}
\hline Erbbauzins in Deutschland & & \\
Umfrage/Bericht & Wohnen (\%) & Gewerbe (\%) \\
\hline Licher (2009) & $3,6-4,0$ & $5,1-5,4$ \\
Aholt und Hartl (2016) & $2,5-3,5$ & 4,0 \\
Grundstücksmarktbericht NRW (2017) & 3,0 & $\mathrm{k} . \mathrm{A}$. \\
Deutscher Erbbaurechtsverband e. V. (2018) & & \\
Neuvergabe & 3,1 & 4,3 \\
Verlängerung & 4,0 & $6,0-7,0$ \\
\hline
\end{tabular}

Entwicklung von unterstützender Software zur Verwaltung von Erbbaurechten beschäftigt. Die Aufstellung erhebt keinen Anspruch auf Vollständigkeit. Ein paar der angeführten Akteure wurden bereits in Löhr $(2017)^{36}$ genannt und sind somit schon länger am Markt aktiv.

Gemäß einer Umfrage des Deutschen Erbbaurechtsverbandes (2018) steigt die Nachfrage nach Erbbaurechten zwar, das Konzept des Erbbaurechts sei aber dem Großteil der Investoren nicht vollumfänglich bekannt. 93\% der Befragten ist der Auffassung, dass Volleigentum einfacher zu vermarkten ist. $74 \%$ der Teilnehmer nutzen das Erbbaurecht nach eigenen Angaben, um einen langfristigen und möglichst sicheren Cashflow zu generieren. Die Teilnehmer der Studie geben an, dass sie auch im kommenden Jahr erneut Erbbaurechte vergeben werden, wobei sich dies oft nur auf die Erneuerung von Altverträgen bezieht.

Erbbauzins Für Investoren interessant ist die Frage nach dem Erbbauzins, da dieser maßgebend für die Rendite und somit auch Höhe eines möglichst sicheren und langfristigen Cashflows ist. Eine Umfrage von Licher (2009) ${ }^{37}$ ordnet die durchschnittliche Höhe im Bereich von 3,6 bis $4 \%$ (für Wohnnutzungen) und 5,1 bis 5,4\% (für gewerbliche Nutzungen) ein. Eine Studie von Aholt und Hartl (2016) ${ }^{38}$ konstatiert 2,5 bis 3,5\% (Wohnen) bzw. $4 \%$ (Gewerbe). Die Befragung des deutschen Erbbaurechtsverbandes (2018) stellt für Wohnimmobilien einen durchschnittlichen Erbbauzins von 3,1\% und für Gewerbeimmobilien von 4,3\% fest (vgl. Tab. 1). Bei Verlängerung oder Neuausgabe des Erbbaurechts erfolgt üblicherweise die Anpassung zu einem höheren Zins. Dieser liegt laut Umfrage bei $4 \%$ für wohnwirtschaftlich genutzte Grundstücke und zwischen 6 und $7 \%$ für Gewerbeflächen (Tab. 2).

Am Markt beobachtete Erbbauzinssätze für Wohnnutzungen verzeichnet der Grundstücksmarkbericht des Bundeslandes Nordrhein-Westfalen (NRW) des Jahres 2017. ${ }^{39}$ Für andere Bundesländer werden diese Angaben nach unserem Kenntnisstand nicht veröffentlicht. Auf Grundlage der Daten von 20 Gutachterausschüssen liegt der Erbbauzins im Bereich individueller Wohnungsbau für im Jahr 2016 abgeschlossene Erbbaurechtsverträge zwischen 1,4 und 4,0\%, im Durchschnitt bei

\footnotetext{
36 Vgl. Löhr (2017).

37 Umfrage mit 126 kommunalen und 20 kirchlichen Teilnehmern, vgl. Licher (2009, S. 52).

38 Vgl. Aholt und Hartl (2016).

39 Vgl. Oberer Gutachterausschuss für Grundstückswerte im Land Nordrhein-Westfalen (2017, S. 31).
} 


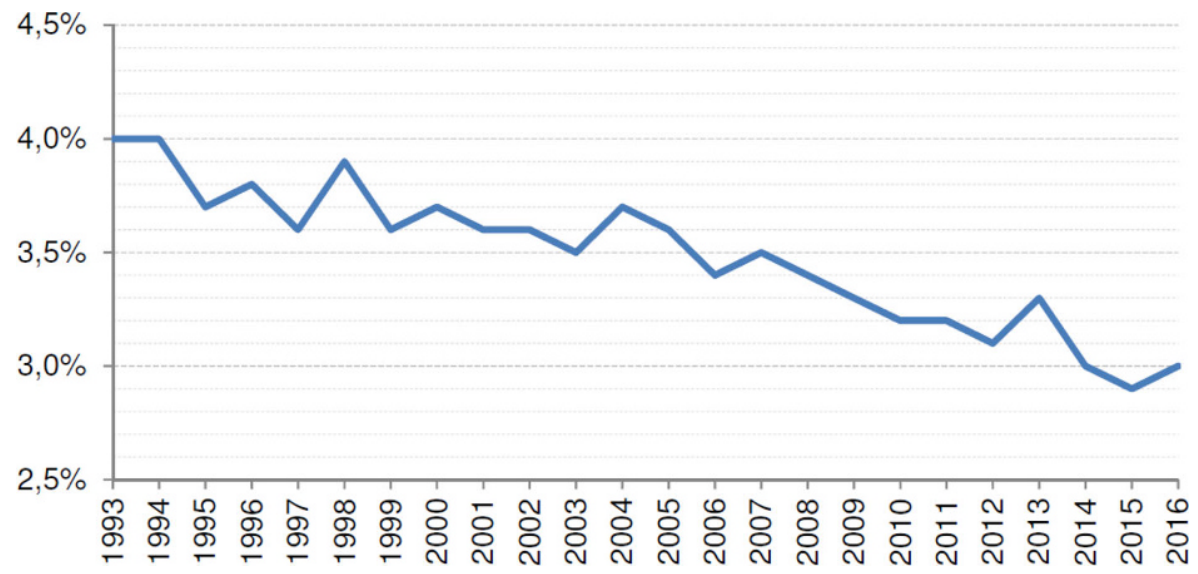

Abb. 1 Entwicklung Erbbauzinssatz NRW. (Oberer Gutachterausschuss für Grundstückswerte im Land Nordrhein-Westfalen 2017, S. 31)

$3,0 \%$. Erbbauzinsen liegen somit deutlich über langfristigen Staats- oder Unternehmensanleihen. Hinzu kommt, dass es sich bei diesen Angaben um Nettorenditen handelt, da keine laufenden Kosten für Instandhaltung o. Ä. anfallen.

Die nachfolgende Grafik zeigt den Verlauf der durchschnittlichen Erbbauzinsen in NRW von 1994-2016 auf (Abb. 1).

Ein Vergleich der Daten aus Nordrhein-Westfalen mit Hypothekenzinsen zeigt, dass Erbbauzinsen nur eine geringe Korrelation mit dem Zinsmarkt aufweisen (vgl. Abb. 2). ${ }^{40}$ Während die Hypothekenzinsen in Deutschland zwischen 1994 und 2016 von 8,8 auf $1,4 \%$ gefallen sind, sank der Erbbauzins im gleichen Zeitraum nur von 4,0 auf 3,0\%. Diese Abkopplung von der Zinsentwicklung gilt nach Löhr (2017) als ein Grund für die momentan geringe Verbreitung von Erbbaurechten. ${ }^{41}$

Für Niedersachen wurden auf Anfrage von der Geschäftsstelle des Oberen Gutachterausschusses die nachfolgenden Darstellungen übermittelt. ${ }^{42}$ Demnach beträgt der Anteil von Erbbaurechten an allen Transaktionen im Durchschnitt etwa 3,4\%. Absolut liegt die Anzahl der Verkäufe zwischen 25.000 und 40.000. Detailliertere Angaben seien gegen Gebühr verfügbar (Abb. 3 und 4).

\subsection{Großbritannien}

Marktvolumen Während für Erbbaurechte an Wohnimmobilien umfassende Informationen vorliegen, existiert für Gewerbeimmobilien keine vergleichbare Datenlage. Die aktuellsten Zahlen der britischen Regierung für Erbbaurechte an Wohnimmobilien umfassen die Jahre 2015 und 2016, allerdings handelt es sich ebenfalls nur um Schätzungen. Demnach gibt es in Großbritannien circa 1,4 Mio. Häuser sowie

\footnotetext{
40 Die Hypothekenzinsen dienen hier zur Veranschaulichung der Zinsentwicklung am Markt für Immobilienfinanzierungen.

41 Vgl. Löhr (2017, S. 4f).

42 Es gab keine Anfrage an andere obere Gutachterausschüsse.
} 


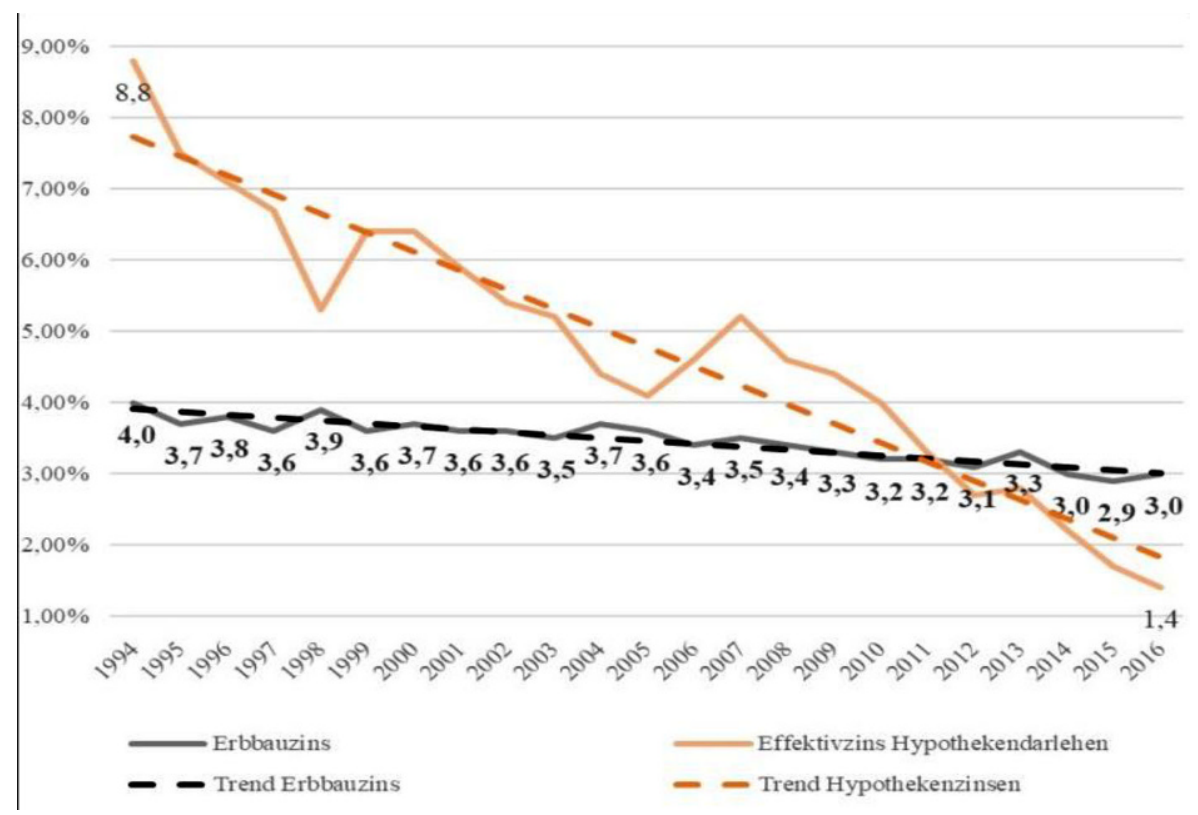

Abb. 2 Entwicklung Erbbauzins in Nordrhein-Westfalen vs. Hypothekenzinsen in Deutschland seit 1994. (Eigene Darstellung nach Daten vom Oberem Gutachterausschuss für Grundstückswerte im Land Nordrhein-Westfalen 2017, S. 31)

Abb. 3 Anteil Transaktionen 2000-2017 zum Erbbaurecht in Niedersachsen

\begin{tabular}{lrr} 
& \multicolumn{2}{c}{$\begin{array}{c}\text { Verkauf des } \\
\text { Erbbaurechts }\end{array}$} \\
\hline $\mathbf{2 0 0 0}$ & ohne Erbbaurecht & $3,20 \%$ \\
$\mathbf{2 0 0 1}$ & $96,80 \%$ & $3,09 \%$ \\
$\mathbf{2 0 0 2}$ & $96,91 \%$ & $3,36 \%$ \\
$\mathbf{2 0 0 3}$ & $96,64 \%$ & $3,38 \%$ \\
$\mathbf{2 0 0 5}$ & $96,62 \%$ & $3,23 \%$ \\
$\mathbf{2 0 0 6}$ & $96,77 \%$ & $3,24 \%$ \\
$\mathbf{2 0 0 7}$ & $96,76 \%$ & $3,55 \%$ \\
$\mathbf{2 0 0 8}$ & $96,45 \%$ & $3,69 \%$ \\
$\mathbf{2 0 0 9}$ & $96,31 \%$ & $3,70 \%$ \\
$\mathbf{2 0 1 0}$ & $96,30 \%$ & $3,83 \%$ \\
$\mathbf{2 0 1 1}$ & $96,17 \%$ & $3,62 \%$ \\
$\mathbf{2 0 1 2}$ & $96,38 \%$ & $3,72 \%$ \\
$\mathbf{2 0 1 3}$ & $96,28 \%$ & $3,52 \%$ \\
$\mathbf{2 0 1 4}$ & $96,48 \%$ & $3,54 \%$ \\
$\mathbf{2 0 1 5}$ & $96,46 \%$ & $3,43 \%$ \\
$\mathbf{2 0 1 6}$ & $96,57 \%$ & $3,48 \%$ \\
$\mathbf{2 0 1 7}$ & $96,52 \%$ & $3,33 \%$ \\
\hline
\end{tabular}




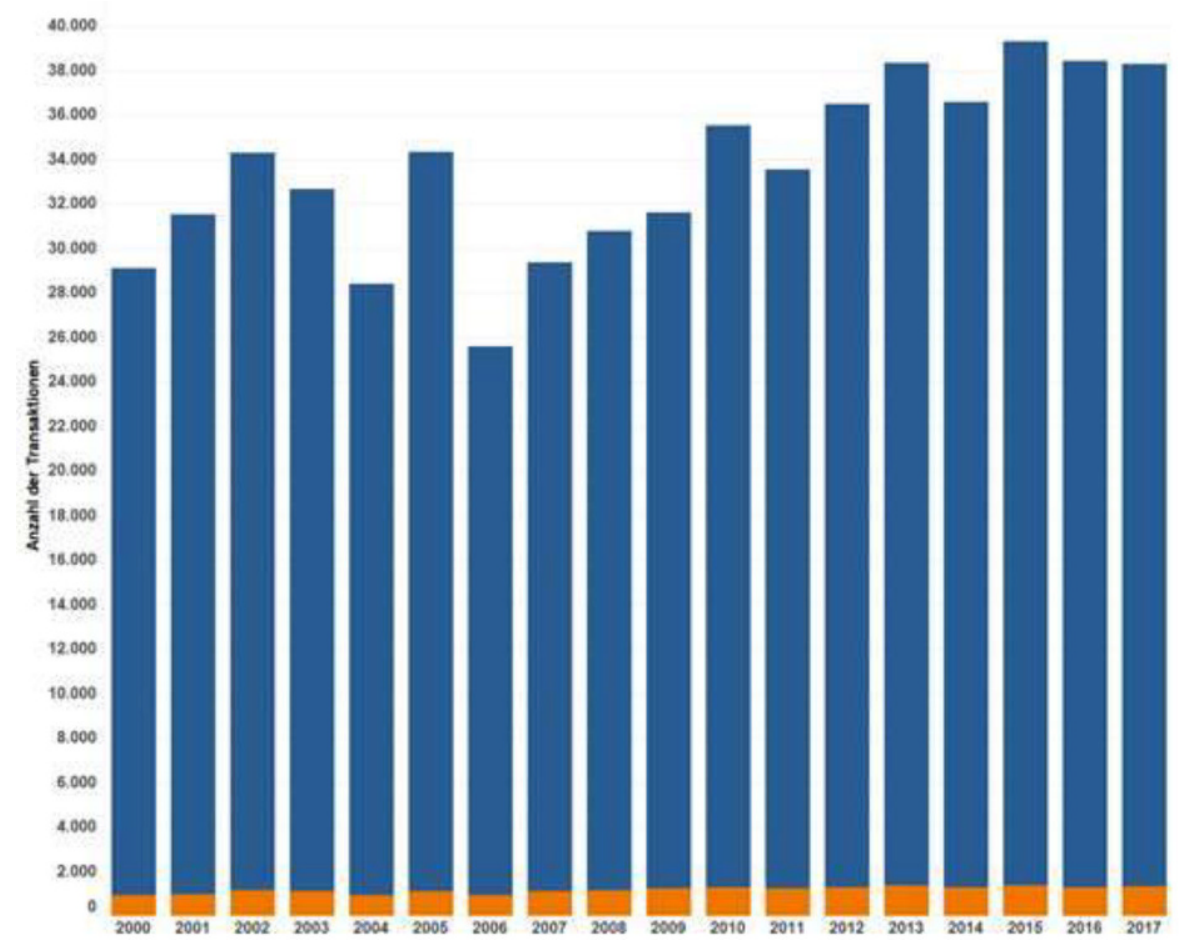

Abb. 4 Anzahl Transaktionen 2000-2017 zum Erbbaurecht in Niedersachsen

2,9 Mio. Wohnungen, die mit einem Leasehold versehen sind. Insgesamt unterliegen in Großbritannien somit rund $18 \%$ aller Immobilien dem Leasehold. Dies entspricht einer Steigerung von $17 \%$ am Häusermarkt respektive $4 \%$ auf dem Wohnungsmarkt im Vergleich zur Vorperiode. Diese Steigerungsraten resultieren vor allem aus der Vergabe von Wohnungen in Großstädten als Leasehold sowie aus der kontrovers diskutierten Beteiligung von Projektentwicklern am Verkauf von Häusern, die dem Leasehold unterliegen. ${ }^{43}$

Eine weitere Regierungsquelle schätzt, dass es gesamt rund vier Millionen Leasehold-Haushalte in England gibt. $70 \%$ davon seien Wohnungen. ${ }^{44}$ Andere Schätzungen bezüglich des Marktvolumens gehen von circa fünf bis sechs Millionen Leasehold-Haushalten aus und liefern auch differenzierte Zahlen dazu, wie sich das Leasehold auf verschiedene Typen aufteilt (Abb. 5). ${ }^{45}$

Eine Untersuchung auf Postleitzahlenebene zeigte, dass manche Bezirke in London und Manchester zu über $90 \%$ aus Leasehold-Eigentum bestehen, während der

\footnotetext{
43 Vgl. Department for Communities and Local Government (2017a).

44 Vgl. Wilson und Barton (2018).

45 Vgl. Studie von The Leasehold Partnership \& Open Data Institute gemäß Wilson und Barton (2018).
} 
Abb. 5 Leasehold-Anteil Immobilienmarkt UK (2017).

(Vgl. Wilson und Barton 2018, S. 11-12)

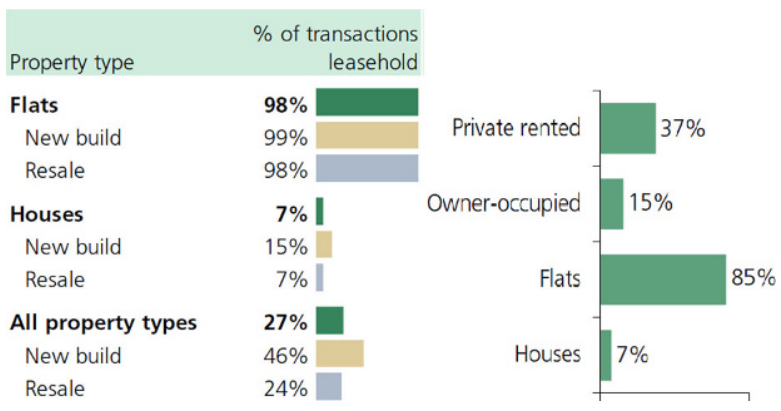

Anteil für England insgesamt zwischen 15 und $18 \%$ liegt. ${ }^{46}$ In den letzten Jahren gab es einen starken Anstieg an neu ausgegebenen Leasehold. Bei Neubauten lag der Anteil in London insgesamt bei $90 \%$. Generell ist festzuhalten, dass sich der Wert eines Grundstücks mit Leasehold an der verbliebenen Vertragsdauer orientiert: Ein Leasehold mit Wohnberechtigung für die nächsten 90 Jahre ist mehr wert als ein Leasehold, das auf 40 Jahre ausgelegt ist. Dies spiegelt sich vor allem in den Verkaufspreisen der Liegenschaft wider.

Savills Research führte 2012 eine Studie zu alternativen Investmentmöglichkeiten durch. Eine dieser alternativen Investmentmöglichkeiten stellt das Leasehold dar. Durch Befragungen von Investoren, die am Markt für Ground Rents aktiv sind, wurden auch Prognosen bezüglich des Kapitalwertes der Ground Rents für die kommenden Jahre erstellt. Aufgrund der aktuellen Änderungen des Leaseholds in Großbritannien sind die Prognosen für das Jahr 2019 anzuzweifeln. Abb. 6 verdeutlicht allerdings auch den hohen Kapitalwert der Ground Rents vergangener Jahre. Zusätzlich zeigt die Grafik auch die Korrelation des Einkommens durch Ground Rent mit der Anzahl der jährlich erstellten Leaseholds, auf.

Ground Rent Im Unterschied zum deutschen Erbbauzins ist der Handel des Cashflows aus Ground Rents erlaubt und war als Fixed Income Investment etabliert. Die Ground Rent ist aber - anders als der deutsche Erbbauzins - nur ein kleiner Teil der zu zahlenden Leistung des Leaseholders. Eine durchschnittliche Ground Rent beträgt circa 50 Pfund pro Monat und wird meist jährlich gezahlt. Der Großteil des Cashflows setzt sich aus der eigentlichen Mietzahlung sowie Servicegebühren und Steuern zusammen.

Die Ausgestaltung der Zahlungsströme für den Grundstückseigentümer lässt sich grundsätzlich in zwei Varianten einteilen: Entweder erhält er zu Beginn der Vertragslaufzeit eine Einmalzahlung („Premium“) und über die Restlaufzeit eine geringe Ground Rent - oder eine monatliche Zahlung ähnlich dem Erbbauzins. ${ }^{47}$ Unabhängig davon erhält der Grundstückseigentümer eine Servicegebühr für beispielsweise Instandhaltungen oder Versicherungen etc. Es existieren nur wenige Studien und

\footnotetext{
46 Vgl. My Home Move Ltd (2016) oder Department for Communities and Local Government (2017a, S. 5).

47 Vgl. Waldner und Kopp (2015), Rn. 50.
} 


\section{GRAPH 2}

\section{Capital Values of New Ground Rents}

- Estimated Capital Value of New Ground Rents a Forecast Capital Value of New Ground Rents

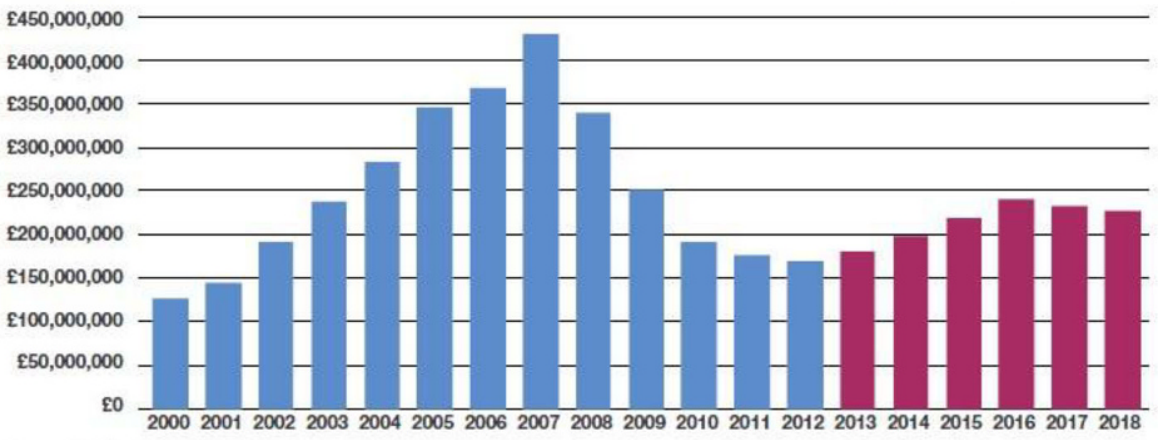

Source: Savills Research

\section{Annual leasehold completions and ground rent income:}

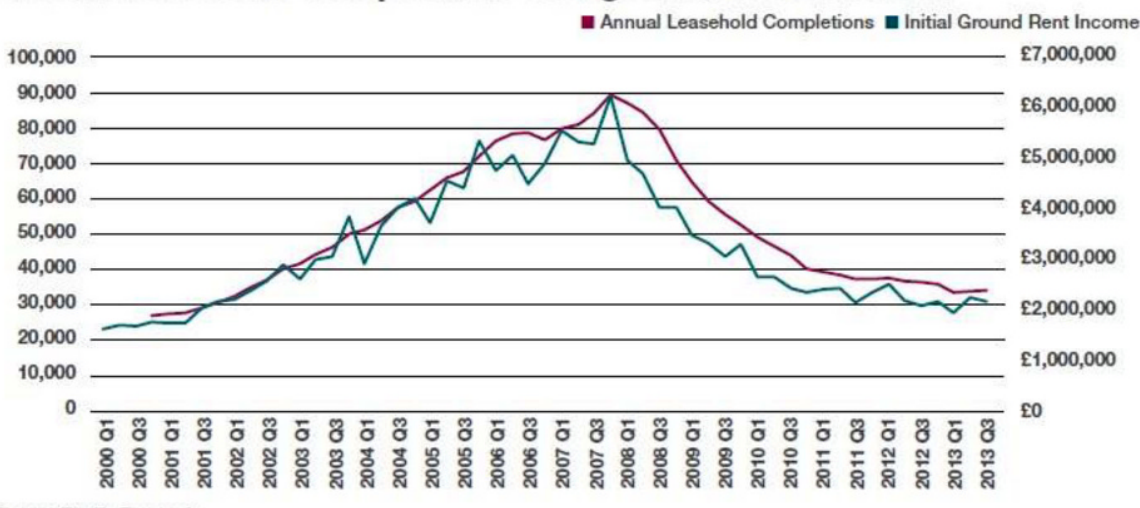

Source: Savills Research

Abb. 6 Übersicht Ground Rent in UK (Savills Research 2014)

Schätzungen zur Ground-Rent. Nach einer Umfrage von Direct Line for Business aus dem Jahr 2016 liegt der Anteil des Leasehold-Nehmers an der Servicegebühr bei durchschnittlich 1863 Pfund pro Jahr. Hinzu kommt die Ground Rent, die jährlich im Durchschnitt 371 Pfund für ein neu errichtetes Haus bzw. 327 Pfund für ein bereits bestehendes Objekt beträgt. Weiterhin können Gebühren und Verpflichtungen wie der Zins einer offenen Hypothek oder auch Steuern hinzukommen. Die Gesamtbelastung des Leaseholder variiert stark je nach Lage der Immobilie. ${ }^{48}$

48 Vgl. Direct Line Insurance Group (2016). 


\section{Investitionsformen}

In der Literatur werden drei Formen der Nutzung des Erbbaurechts durch institutionelle Investoren unterschieden: ${ }^{49}$

- Direktinvestment: Der Investor hält individuelle Kapitalanlagen, die mit dem Erbbaurecht belastet sind, direkt in der Bilanz.

- Fonds: Der Anleger investiert in eine Fondsstruktur, um den Zugang zu einem Portfolio bestehend aus Anlagen im Bereich Erbbaurecht zu erhalten. Die Anzahl der einzelnen Einheiten, die im Fonds gehalten werden, definiert den Anteil jedes zugrundeliegenden Assets, zu welchem der Investor Zugang hat.

- Besicherte Anleihe/Schuldverschreibung: Die dritte Möglichkeit beschreibt den Kauf einer Anleihe beziehungsweise einer Schuldverschreibung, welche mit dem langfristigen Cashflow des Erbbauzinses besichert ist.

\subsection{Immobilien-Spezialfonds mit Fokus Erbbaurecht}

In Großbritannien existiert eine Reihe von Immobilien-Spezialfonds mit dem Fokus Erbbaurecht. Einer der größten Fonds ist gemäß eigener Angabe der Long Harbour Ground Rent Fund mit Assets under Management in Höhe von 1,4 Mrd. Pfund. Der Fokus des Fonds liegt im wohnwirtschaftlichen Bereich. Der Fonds existiert seit dem Jahr 2010 und hat bisher über 160.000 Wohneinheiten, die mit einer Ground Rent belastet sind, erworben. Das Geschäftsmodel sieht vor, Portfolien im Wert von 20.000 bis 240 Mio. Pfund zu erwerben. Diese Portfolien bestehen zumeist aus dem Freehold an Grundstücken, für welche anschließend die Vergabe des Leasehold erfolgt. Dabei ist die ausstehende Laufzeit des jeweiligen Ground-Rent-Vertrages uninteressant. Die Verwaltung der angekauften Erbbaurechtsgrundstücke sowie die Betreuung des Leaseholders werden von einer externen Gesellschaft übernommen. ${ }^{50}$

Auch im gewerblichen Bereich existieren große Fonds wie der Index Linked Income Fund von Alternative Real Capital mit einem Volumen von über einer Milliarde Pfund. Der Fonds investiert einerseits in bereits existente Ground Leases, schafft andererseits aber auch selbst neue Ground Leases. Bei bestehenden Verträgen wird auf eine möglichst lange Restlaufzeit geachtet (typischerweise 99 Jahre). Zusätzlich werden primär Ground Leases mit Inflationsindexierung in das Portfolio aufgenommen. Des Weiteren wird versucht, Synergien mit Projektentwicklern in Form von Forward Commitment Arrangements zu erreichen (Tab. 3). ${ }^{51}$

In Deutschland ist das Produkt Erbbaurecht in der Fondsindustrie derzeit nicht etabliert. Der Erbbauzins Deutschland Core Fonds (Auflagedatum: 2011) der Arvbo Erbbau AG, Hamburg sowie der 2004 aufgelegte Deutsche Wohnungsprivatisierungs-Fonds Nr. 1 (DWF1) waren unseres Wissens nach die einzigen Fonds in Deutschland, die sich auf Erbbaurechte fokussiert hatten. Die Arvbo Erbbau AG hat über IntReal einen Spezialfonds für institutionelle Anleger aufgelegt. Die

\footnotetext{
49 Institute und Faculty of Actuaries (2015).

50 Vgl. Long Harbour Ltd (2018).

51 Vgl. Alternative Real Capital (2018).
} 
Tab. 3 Erbbaurechtsfonds in Großbritannien

\begin{tabular}{|c|c|c|c|c|}
\hline Unternehmen & Produktname & Auflage & $\begin{array}{l}\text { Volumen } \\
\text { in GBP }\end{array}$ & Objective \\
\hline $\begin{array}{l}\text { Long Harbour } \\
\text { Ltd }\end{array}$ & $\begin{array}{l}\text { Long Harbour Ground } \\
\text { Rent Fund }\end{array}$ & 2010 & $1,4 \mathrm{Mrd}$ & Residential ground rents \\
\hline $\begin{array}{l}\text { Alternative Real } \\
\text { Capital }\end{array}$ & $\begin{array}{l}\text { Index Linked Income } \\
\text { Fund }\end{array}$ & $02 / 2016$ & $1 \mathrm{Mrd}$ & Commercial ground rents \\
\hline $\begin{array}{l}\text { Pramerica Real } \\
\text { Estate Investors }\end{array}$ & $\begin{array}{l}\text { PGIM Real Estate UK } \\
\text { Ground Lease Fund }\end{array}$ & $02 / 2007$ & $641,7 \mathrm{Mio}$ & $\begin{array}{l}\text { Residential and commercial } \\
\text { ground rents }\end{array}$ \\
\hline Standard Life & $\begin{array}{l}\text { Standard Life Com- } \\
\text { mercial Ground Rent } \\
\text { Pension Fund }\end{array}$ & $03 / 2012$ & 291 Mio & $\begin{array}{l}\text { Retail, office, industrial and } \\
\text { other commercial sectors }\end{array}$ \\
\hline $\begin{array}{l}\text { Alternative Real } \\
\text { Capital }\end{array}$ & Social Long Income Fund & $05 / 2017$ & 5,2 Mio & $\begin{array}{l}\text { Social real estate and infra- } \\
\text { structure }\end{array}$ \\
\hline
\end{tabular}

Rechtsform wird nicht angegeben. Nach Unternehmensangaben von 2012 war ein Zielvolumen von 100Mio. $€$ und eine Ausschüttung von 4-4,5\% geplant. Primär soll es sich dabei um Wohnimmobilien handeln. ${ }^{52}$ Auf Anfrage an die Arvbo Erbbau AG teilte diese mit, dass keine weiteren Informationen herausgegeben werden, da dieser Fonds derzeit nicht mehr im Vertrieb sei. Auch der Vertrieb des DWF1 wurde innerhalb eines Jahres, aufgrund schleppender Verkäufe an Privatinvestoren, eingestellt.

\subsection{Fremdkapital-Produkte}

Großbritannien Die erste mit Ground Rent besicherte Anleihe kam im März 2006 auf den Markt. ${ }^{53}$ Emittent war die Fairhold Securitisation Limited. Im Jahr 2015 kam es zu vermehrten Ausfällen aus den Ground Rents, wodurch die Käufer der Anleihe keine Zahlungen mehr erhielten. Die Verbindlichkeiten an die Anteilseigner waren zwar durch Swaps abgesichert, es kam aber zu einem Rechtsstreit mit der ausgebenden Gesellschaft UBS. Durch Absicherung mittels Swaps war es möglich, bis Oktober 2015 zu überleben. ${ }^{54}$ Die Anleihe hatte eine Laufzeit bis Oktober 2017. Die weitere Entwicklung ist uns nicht bekannt (Tab. 4).

Deutschland Bei den wenigen Verbriefungstransaktionen, die in Deutschland stattgefunden haben, handelt es sich um CMBS in Kombination mit Schuldscheinen mit mehrjähriger Laufzeit der Vivacon AG. Die Verbriefungen hatten Laufzeiten von 7 bis zu 27 Jahren. ${ }^{55}$ Die Verträge der Erbbaurechtsgrundstücke, die im Portfolio enthalten sind, weisen dabei typischerweise lange Restlaufzeiten auf. Die Angaben zu Rating und Volumina beziehen sich auf die aktuellsten externen Beurteilungen des Vehikels. Weitere Angaben wurden aus den jeweiligen Pressemitteilungen so-

\footnotetext{
52 Vgl. Private Banking Magazin (2012).

53 Der deutsche Markt wird im diesem Zusammenhang als Vorreiter für dieses Produkt bezeichnet, vgl. GlobalCapital (2006). Das Produkt hat sich jedoch auch in Deutschland nicht etabliert.

54 Vgl. McNeill und Williamson (2017).

55 Vgl. Hansen (2006).
} 
Tab. 4 Ground Rent ABS-Anleihe in Großbritannien

\begin{tabular}{|c|c|c|c|c|c|c|}
\hline Unternehmen & Produktname & Art & Auflage & Volumen & Rating & $\begin{array}{l}\text { Projektierte } \\
\text { Rendite }\end{array}$ \\
\hline $\begin{array}{l}\text { Fairhold Securi- } \\
\text { tisation Ltd }\end{array}$ & $\begin{array}{l}\text { Second Issue } \\
\text { Further Class A } \\
\text { Notes }\end{array}$ & $\begin{array}{l}\text { ABS- } \\
\text { Anlei- } \\
\text { hen }\end{array}$ & $\begin{array}{l}\text { März } \\
2006\end{array}$ & $\begin{array}{l}\text { 84,7 Mio. } \\
€\end{array}$ & AAA & $\begin{array}{l}6 \mathrm{M} \text { LIBOR } \\
+85,5 \mathrm{bps}\end{array}$ \\
\hline $\begin{array}{l}\text { Fairhold Securi- } \\
\text { tisation Ltd }\end{array}$ & $\begin{array}{l}\text { Second Issue } \\
\text { Further Class B } \\
\text { Notes }\end{array}$ & $\begin{array}{l}\text { ABS- } \\
\text { Anlei- } \\
\text { hen }\end{array}$ & $\begin{array}{l}\text { Mai } \\
2007\end{array}$ & $\begin{array}{l}\text { 5,8 Mio. } \\
€\end{array}$ & Baa2 & $\begin{array}{l}6 \mathrm{M} \text { LIBOR } \\
+125 \mathrm{bps}\end{array}$ \\
\hline
\end{tabular}

Tab. 5 Erbbaurecht ABS-Anleihe in Deutschland

\begin{tabular}{|c|c|c|c|c|c|c|}
\hline $\begin{array}{l}\text { Unter- } \\
\text { nehmen }\end{array}$ & Produktname & Art & Auflage & Volumen & Rating & Projektierte Rendite \\
\hline $\begin{array}{l}\text { Vivacon } \\
\mathrm{AG}\end{array}$ & $\begin{array}{l}\text { German } \\
\text { Ground Lease } \\
\text { Finance } 1\end{array}$ & $\begin{array}{l}\text { ABS- } \\
\text { Anlei- } \\
\text { hen }\end{array}$ & $\begin{array}{l}\text { März } \\
2006\end{array}$ & $\begin{array}{l}62 \text { Mio. } \\
€\end{array}$ & Baa1 & $4,74 \%$ (Kupon) \\
\hline $\begin{array}{l}\text { Vivacon } \\
\mathrm{AG}\end{array}$ & $\begin{array}{l}\text { German } \\
\text { Ground Lease } \\
\text { Finance } 2\end{array}$ & $\begin{array}{l}\text { ABS- } \\
\text { Anlei- } \\
\text { hen }\end{array}$ & $\begin{array}{l}\text { Dez. } \\
2006\end{array}$ & $\begin{array}{l}\text { 122,8 Mio. } \\
€\end{array}$ & AAA & $\begin{array}{l}\text { 1: } 3 \mathrm{M} \text { Euribor }+22 \mathrm{bps} \\
\text { 2: } 3 \mathrm{M} \text { Euribor }+33 \mathrm{bps} \\
\text { (Kupon) }\end{array}$ \\
\hline $\begin{array}{l}\text { Vivacon } \\
\mathrm{AG}\end{array}$ & $\begin{array}{l}\text { German } \\
\text { Ground Lease } \\
\text { Finance } 3\end{array}$ & $\begin{array}{l}\text { ABS- } \\
\text { Anlei- } \\
\text { hen }\end{array}$ & $\begin{array}{l}\text { Nov. } \\
2008\end{array}$ & $\begin{array}{l}90 \text { Mio. } \\
€\end{array}$ & AAA & $\begin{array}{l}\text { 1: } 3 \mathrm{M} \text { Euribor }+113,8 \mathrm{bps} \\
\text { 2: } 3 \mathrm{M} \text { Euribor }+180 \mathrm{bps} \\
\text { (Kupon) }\end{array}$ \\
\hline
\end{tabular}

wie den Fact Sheets entnommen. Seit der Insolvenz der Vivacon AG im Jahr 2014 verwaltet die Immofori AG deren Anleihen. Tab. 5 gibt eine Übersicht über alle uns bekannten Emissionen.

\subsection{Exkurs: Erbbaurecht im Kontext von Solvency II}

Die Kapitalvoraussetzung gemäß Solvency II hängt von der Art des Investments ab. Einzelne Erbbaurechte können dabei bis zu $10 \%$ des Sicherungsvermögens ${ }^{56}$ ausmachen. Eine Direktinvestition in Erbbaurechtsgrundstücke wird als direktes Halten von Immobilien eingestuft, wodurch $25 \%$ Eigenkapital vorgehalten werden müssen. Anleihen haben den Vorteil, dass der zugrundeliegende Cashflow, der aus dem Erbbauzins beziehungsweise der Ground Rent stammt, vom Risiko der vorzeitigen Tilgung befreit wird. Dadurch werden die Voraussetzungen für die Matching-Anpassung erfüllt. Das Investment kann somit laut Solvency II als Bond behandelt werden. Bei einem nicht bewerteten Bond mit 30 Jahren Laufzeit könnte dies zu Eigenkapitalunterlegung von $20,25 \%$ führen. ${ }^{57}$

Um die Kapitalanforderung nach Solvency II zu berechnen, wird entweder auf die Standardformel oder auf interne modelbasierte Ansätze zurückgegriffen. Letztere greifen nicht zwangsweise auf vorgegebene Stressfaktoren und Ratings zurück, sondern erlauben die eigene Einschätzung des Risikos des jeweiligen Assets. Erb-

\footnotetext{
56 Sicherungsvermögen bezeichnet das vom Versicherungsunternehmen für den Versicherungsnehmer investierte Vermögen.

57 Vgl. Institute und Faculty of Actuaries (2015, S. 79).
} 
baurechte unterliegen typischerweise keinem Rating und weisen sehr lange Laufzeiten auf. Dies führt laut Standardansatz, selbst mit Matching-Anpassung ${ }^{58}$, zu vergleichsweise hohen Kapitalanforderungen. Mittels interner Ansätze ist es möglich, dem geringen Risiko des Erbbaurechts Rechnung zu tragen, womit geringere Kapitalanforderungen möglich sind. Problematisch ist die mangelnde Verfügbarkeit von langen Datenhistorien im Bereich der Erbbaurechte. Ohne entsprechende Zeitreihe können weder Kredit- noch Liquiditätsrisiko richtig eingeschätzt werden. Dies wird für interne Modellierungen benötigt, um das Spreadrisiko richtig einzuordnen. Gleiches trifft auf die in England bekannte Ground Lease zu. ${ }^{59}$

\section{Verfahren zur Wertermittlung}

Die Beleihungswertermittlung erfolgt in der Praxis meist durch inländische Verfahren, wie das Münchner Verfahren. Dabei handelt es sich um das gängigste Verfahren zu Ermittlung des Beleihungswertes in Deutschland. Falls ein Objekt beleihbar ist, ist selbigem auch ein Wert beizumessen, was für die Marktfähigkeit und die Akzeptanz essenziell ist. Eine Verkehrswertermittlung stützte sich bisher auf die Vorgaben der WertR, die in der ImmoWertV aufgegangen ist. Sofern diese Ermittlung standardisiert ist, wird sie auch transparent für die Marktteilnehmer. Aus seiner Eigenschaft als grundstücksgleiches Recht folgt, dass das Erbbaurecht wie ein Grundstück mit Hypotheken oder Grundpfandrechten belastet werden kann.

\subsection{Beleihungswert gemäß Münchner Verfahren}

Dabei wird das Erbbaurecht zunächst ausgeblendet und ein regulärer Beleihungswert aus fiktiven Volleigentum bestimmt. So ein Wert lässt sich beispielsweise durch Vergleichswerte ermitteln. Nachgelagert werden drei Schritte durchgeführt:

1. Zeitadäquate Wertabschläge werden durch das Erbbaurecht einberechnet. Dies erfolgt durch Kapitalisierung des Erbbauzinses auf den Bodenwert, bezogen auf die Zeit nach Ablauf des Erbbaurechtes, um den Wegfall des Bodennutzungsrechts einzubeziehen. ${ }^{60}$

2. Zusätzlich berichtigt wird um einen möglichen nicht abzulösenden Gebäudewert am Ende der Laufzeit durch den Erbbaurechtsgeber sowie

3. Individuelle Vertragsbestimmungen, die wiederum aufgrund ihrer Komplexität mit einem Pauschalabschlag berücksichtigt werden.

\footnotetext{
58 Um die Matching-Anpassung der Solvency 2 Verordnung nutzen zu können müssen gewisse Auflagen vom Asset erfüllt werden. So muss es sich beispielsweise um ein Asset mit fixem Cashflow handeln. Erbbaurechte erfüllen normalerweise diese sowie auch weitere Anforderungen.

59 EY jedoch gibt an, interne Modelle entwickelt zu haben, die ohne lange Zeitreihen implementiert werden können, vgl. Ernst und Young (2017).

60 Vgl. Ingold (2017).
} 
In Summe erhält man den Beleihungswert des erbbauzinsfreien Bodenwerts. Die BaFin bestätigt die Validität dieses Verfahrens - zumindest, sofern Mindestabschläge bzw. Höchstkapitalisierungsgrenzen eingehalten werden. ${ }^{61}$

Für die Beleihung, die für die letztendliche Finanzierung ausschlaggebend ist, ist neben der Beleihungsgrenze auch noch die Rangstelle relevant. Dabei sind zwei Grundbücher zu unterscheiden, die des Grundstücks und die des Erbbaurechts. So besteht ein Vertrag zwischen den Eigentümern des Erbbaurechts und des originären Grundstückseigentümers. Das Erbbaurecht wird in Abteilung II per Gesetz an 1. Rangstelle eingetragen. Eine nachrangige Stelle des Erbbaurechts bedarf der Zustimmung des ersten Ranges. ${ }^{62}$ Der zu entrichtende Erbbauzins als Reallast findet sich wiederum jedoch in Abteilung II des Erbbaugrundbuches. Da der dingliche Erbbauzins demnach nicht Inhalt des Erbbaurechts ist, gibt es nach $\$ 9$ ErbbauRG Spielraum für die Rangstelle und somit sind grundsätzlich drei Konstellationen möglich, die die Beleihungsgrenze und somit das Fremdfinanzierungsvolumen beeinflussen. Dingliche Vorlasten sind dabei grundsätzlich von der Beleihungsgrenze abzuziehen. Eine Ausnahme hiervon liegt vor, falls

1. der Erbbauzins im Nachrang ohne Vereinbarung zum Bestehenbleiben des Erbbaurechts im Zwangsversteigerungsfall eingetragen ist. Dann bleibt die Beleihungsgrenze des Erbbaurechts unberührt vom Erbbauzins.

2. der Erbbauzins im Vorrang ohne eine Vereinbarung eingetragen ist, muss er in kapitalisierter Form von der Beleihungsgrenze in Abzug gebracht werden.

3. es eine solche Vereinbarung für den Zwangsversteigerungsfall gibt, ist der Erbbauzins vom Beleihungswert in Abzug zu bringen.

Zusammenfassend lässt sich festhalten, dass die konkrete Ausgestaltung der Grundbucheintragungen verschiedene Beleihungsgrenzen erzeugt. Die Variante ohne Vereinbarung mit Nachrangigkeit würde ceteris paribus günstigere Zinskonditionen induzieren. Daneben muss auch beachtet werden, dass die planmäßige Tilgung eines Darlehens durch eine maximale Laufzeit, gleich der Erbbaurechtslaufzeit abzüglich 10 Jahre, nach oben hin limitiert ist. Einer gewissen Subjektivität sind die Abschläge aufgrund allgemeiner Nachteile aus dem Erbbaurechtsvertrag ausgesetzt. Diese Abschläge werden typischerweise nach gutachterlichem Ermessen anhand vorhandener Erfahrungswerte angesetzt. Dabei obliegt es dem Gutachter, wie stark die jeweilige Einschränkung durch das auferlegte Erbbaurecht zu gewichten ist. Schlussendlich lässt sich festhalten, dass das Münchner Verfahren zu einem angemessenen Wert führt, wenn der Pauschalabzug im 3. Schritt marktkonform abgeleitet werden könnte. ${ }^{63}$

Fallbeispiele zur Bewertung mit Hilfe des Münchner Verfahrens sind unter anderem in der vorher benannten Studie zum Thema Erbbaurecht von Jones Lang LaSalle oder Ingold (2017) aufgeführt. ${ }^{64}$

\footnotetext{
${ }^{61}$ Vgl. Bundesanstalt für Finanzdienstleistungen (BaFin) (2009).

${ }^{62}$ Vgl. Meusel (2017, S. 26).

63 Vgl. Ingold (2017, S. 7).

${ }^{64}$ Vgl. Jones Lang LaSalle (2019); vgl. Ingold (2017).
} 


\subsection{Verkehrswert nach ImmoWertV}

Die Ermittlung des Verkehrswertes des Erbbaurechtes sowie des mit dem Erbbaurecht belasteten Grundstücks kann nach den Verfahren der ImmoWertV erfolgen. Theoretisch ist es möglich, dass sich durch die Schaffung eines Erbbaurechts ein Mehrwert gegenüber dem Verkehrswert des Volleigentums ergibt. Folgendes Beispiel illustriert das Vergleichswertverfahren (Tab. 6). ${ }^{65}$

Zur Ermittlung des Verkehrswertes des Erbbaurechts wird ein Vergleichsfaktor i.H.v. 0,85 herangezogen, der auf Transaktionen vergleichbarer Objekte mit und ohne Erbbaurecht basiert. Aufgrund dieses Faktors ergibt sich der Verkehrswert des Erbbaurechtes von ca. $140.000 €$, berechnet aus dem Verkehrswert des unbelasteten bebauten Grundstücks i.H.v. $165.000 €$ multipliziert mit 0,85 . Die zweite Komponente, der Verkehrswert des belasteten Erbbaurechtsgrundstücks, ergibt sich gemäß Vergleichswertverfahren aus der Multiplikation des Bodenwertes des unbelasteten Grundstücks $(60.000 €)$ mit einem möglichen Vergleichsfaktor von 0,7. Der Verkehrswert des Erbbaurechtsgrundstücks wird somit auf $42.000 €$ geschätzt. Vergleichsfaktoren werden von Gutachterausschüssen, spezifiziert für den jeweiligen Teilmarkt, bestimmt.

Zusammengefasst ergibt sich nach diesem Beispiel ein Gesamtwert i.H.v. $182.000 €$. Somit könnte hier durch die erbbaurechtliche Aufspaltung des Grundstückes gegenüber dem Volleigentum ein Mehrwert von $17.000 €$ geschaffen werden. Sofern sich am Markt andere Vergleichsfaktoren ergeben, ist entsprechend auch ein Mindererlös möglich.

$\mathrm{Zu}$ erwähnen ist allerdings, dass das finanzmathematische Verfahren aufgrund fehlender Vergleichsfaktoren häufiger Einsatz findet. ${ }^{66}$ Die Verkehrswertermittlung des Erbbaurechtes gemäß finanzmathematischer Methode erfolgt folgendermaßen: Zunächst wird der Bodenwertanteil des Erbbaurechtes berechnet. Dieser ergibt sich aus der kapitalisierten Differenz von Liegenschaftszins und Erbbauzins bezogen auf Restlaufzeit und zugrundeliegendem Liegenschaftszins. Der Verkehrswert des Erbbaurechtes bestimmt sich aus dem Sachwert des Gebäudes zuzüglich des berechneten Bodenwertanteils und eines Marktanpassungsfaktors. Hinzukommen können auch noch mögliche $\mathrm{Zu}$ - und Abschläge für diverse vertragliche Vereinbarungen.

Tab. 6 Beispiel Verkehrswert nach ImmoWertV

\begin{tabular}{ll}
\hline Bodenwert des unbelasteten unbebauten erschließungsbeitragsfreien Grundstücks & $60.000 €$ \\
Verkehrswert des unbelasteten bebauten Grundstücks & $165.000 €$ \\
Sachwert der baulichen Anlagen & $100.000 €$ \\
Restnutzungsdauer der baulichen Anlagen & 50 Jahre \\
Restlaufzeit des Erbbaurechtsvertrags & 50 Jahre \\
Jährlich erzielbarer Erbbauzins (wertgesichert) 3,0\% & $748,95 €$ \\
Verzinsungsbetrag des Bodenwerts (Liegenschaftszins) & $1800 €$ \\
\hline
\end{tabular}

65 Vgl. Bundesministerium für Umwelt, Naturschutz, Bau \& Reaktorsicherheit (2006).

66 Für weitere Informationen siehe Kleiber (2017, S 2961 ff.) 
Der Verkehrswert des Erbbaugrundstücks gemäß finanzmathematischem Verfahren ergibt sich aus der Summe des abgezinsten Bodenwertes und dem Barwert des vertraglich vereinbarten Erbbauzinses. Auch hier ergeben ein Marktanpassungsfaktor sowie mögliche $\mathrm{Zu}$ - und Abschläge aufgrund vertraglicher Vereinbarungen den Verkehrswert des Erbbaugrundstücks.

Zusammengefasst ergibt sich ein Verkehrswert des Erbbaugrundstücks von $46.000 €$ sowie der Verkehrswert des Erbbaurechts von $140.000 €$, somit ein Gesamtwert von $186.000 €$. Wie im Fall der Bewertung nach Vergleichswertverfahren ist auch hier ein potenzieller Mehrwert von $21.000 €$ geschaffen worden. Auch hier kann es zu einem Mindererlös kommen, sofern sich die Marktanpassungsfaktoren ändern.

\subsection{Marriage Value}

Im Unterschied dazu ist in Großbritannien das Verfahren des Marriage- und Hope Value gängig. Das Konzept des Marriage Value beschreibt den Wertzuwachs bei Vereinigung von Leasehold und Freehold. Das Konzept wurde in Großbritannien entwickelt, ist aber grundsätzlich auf alle Erbbaurechte anwendbar. Allgemein ist der Marriage Value in der Immobilienbewertung nach Definition der European Valuation Standards ,ein höherer Wert, der sich ergibt, wenn der Gesamtwert mehrerer Immobilien (bzw. mehrerer Rechtspositionen am gleichen Objekt) zusammen höher ist als der Wert der Summe aller Bestandteile. " ${ }^{67}$ Deutsche Bezeichnungen wären in etwa Verschmelzungs- oder Synergiewert.

Im hier betrachteten Zusammenhang entsteht ein Marriage Value dadurch, dass der Wert des unbelasteten Freehold höher ist als die Summe aus dem Wert des Leasehold und dem Wert des belasteten Freehold. ${ }^{68}$ Dies impliziert folgende Gleichung:

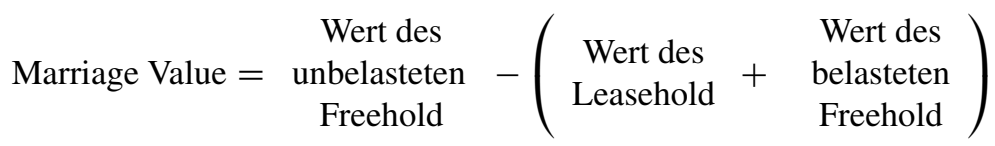

Der auch als Vacant Possession Value bekannte Wert des (hypothetisch) unbelasteten Freehold wird mit den üblichen Verfahren der Immobilienbewertung ermittelt, vorzugsweise anhand von Vergleichstransaktionen. Der Wert des Leasehold ergibt sich als ein prozentualer Anteil am Marktwert des unbelasteten Freehold. Allgemein liegt er zwischen einem Wert nahe $100 \%$ für noch sehr lange laufende Leaseholds und sinkt bei kurzen Restlaufzeiten auf $0 \%$.

Zur Ermittlung des genauen Prozentsatzes kommen in der englischen Bewertungspraxis die sogenannten Graphs of Relativity zum Einsatz. Diese ordnen der noch vorhandenen Restlaufzeit eines Leaseholds jeweils einen zum Wert des unbelasteten Freehold relativen Prozentsatz zu. ${ }^{69}$ In der Praxis werden diverse Graphen und Modelle verwendet, die sich je nach veröffentlichender Institution und betrach-

\footnotetext{
67 TEGOVA - The European Group of Valuers' Associations (2016), EVS 2, Gliederungspunkt 5.3.2.

68 Vgl. Eisenhauer (1997, S. 82).

69 Vgl. Grover (2014, S. 643-651).
} 


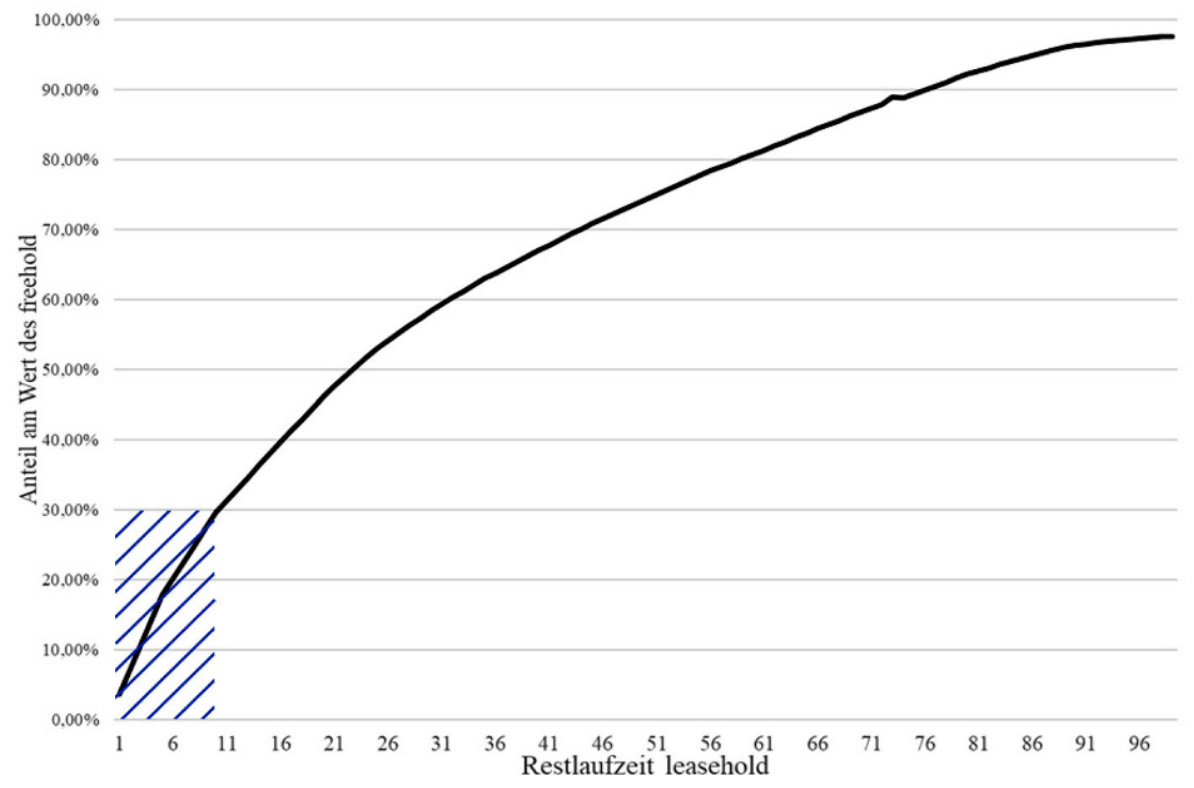

Abb. 7 Relativitätsgraph

tetem Markt unterscheiden. Einen beispielhaften Verlauf zeigt die untenstehende Abb. 7. Der Wert des belasteten Freehold berechnet sich schließlich aus dem kapitalisierten Cashflow der Ground Rent sowie dem Wert des Freehold nach Ablauf des Leaseholds, das als Freehold Reversion bezeichnet wird..$^{70}$ Dieser Ansatz findet sich auch im Münchner Verfahren wieder.

Ein Marriage Value kann durch die Vereinigung von Leasehold und Freehold in einer Hand erzielt werden, aber auch durch Verlängerung eines bestehenden Leasehold hin zu einer sehr langen Restlaufzeit. Bei Astberrys (2009) entsteht der Marriage Value etwa durch Verlängerung eines noch 70 Jahre laufenden Leasehold um weitere 90 Jahre. $^{71}$ Maßgeblich dafür, ob ein Marriage Value entsteht, ist eine Vereinigung der Kapitalinteressen der Eigentümer von Freehold und Leasehold. ${ }^{72}$

Der Grund der Entstehung eines Marriage Value erklärt sich durch die Betrachtung der zugrundeliegenden Kapitalwertberechnung. ${ }^{73}$ Eine Werterhöhung kann bei Anwendung der Kapitalwertmethode entweder durch eine Veränderung der Ein- und Auszahlungshöhe oder durch einen niedrigeren Kapitalisierungszinssatz zustande kommen. An der Höhe der Ein- und Auszahlungen ändert sich durch den Erwerb des Freeholds durch den Leasehold-Eigentümer nichts. Auch die jährliche Ground Rent bleibt unverändert bestehen. Der Marriage Value muss also durch das Sinken des Kapitalisierungszinssatzes entstehen. Dieser sinkt zum einen dadurch, dass ein

\footnotetext{
70 Vgl. Eisenhauer (1997, S. 103).

71 Vgl. Astberrys (2009, S. 1f.).

72 Vgl. Eisenhauer (1997, S. 103 f.).

73 Vgl. Baum et al. (2018, S. 192).
} 
Freehold ohne Belastung als riskanter gilt als derselbe Freehold mit einer auf ihm ruhenden Belastung durch einen Leasehold. Zum anderen sinkt der Kapitalisierungszins auch in Fällen wie bei Astberrys (2009), wenn der Leasehold zwar bestehen bleibt, dieser allerdings zeitlich deutlich ausgedehnt wird. Dies ist der Fall, weil am Markt kurzfristig laufende Leases als riskanter gelten als solche mit längerer Laufzeit. Daraus folgt, dass das Phänomen des Marriage Value vor allem bei Leaseholds mit kurzer Laufzeit zu beobachten ist. ${ }^{74}$

\subsection{Konzept „Hope Value“6}

Der sogenannte Hope Value beschreibt die Möglichkeit, den Mehrwert in Form des Marriage Value durch das Halten beider Positionen zu erzielen. Als Bestandshalter könnte man in die Position des Erbbaurechtsnehmers treten, um das Objekt gewerblich oder wohnwirtschaftlich zu vermieten und im Gegenzug zu dem Nutzen der Mieterträge die Erbbauzinsen bedienen, die i.d.R. nur einen Bruchteil davon ausmachen. Auch wenn dies die Beleihungsgrenze reduziert, ist ein stabiler und grundsätzlich sicherer Cashflow gewährleistet, inklusive Liquiditätsvorteil. ${ }^{75}$ Zusätzlich „hofft“ der Bestandshalter darauf, vor Ablauf der regulären Laufzeit das Grundstück zu erwerben. Somit würde er den Wert des belasteten Grundstücks nebst Nebenkosten leisten, erhält aber dadurch den Wert des unbelasteten Grundstücks inklusive Marriage Value. Es existieren optimale Erwerbsbereiche, die den Marriage Value maximieren. Falls es nicht zu einem vorzeitigen Aufkauf kommt, erhält der Erbbaurechtsnehmer nach britischem Recht jedoch eine Prolongation bzw. im Zweifel in jedem Fall eine Ausgleichzahlung, was das Spekulationsrisiko limitiert (Abb. 8).

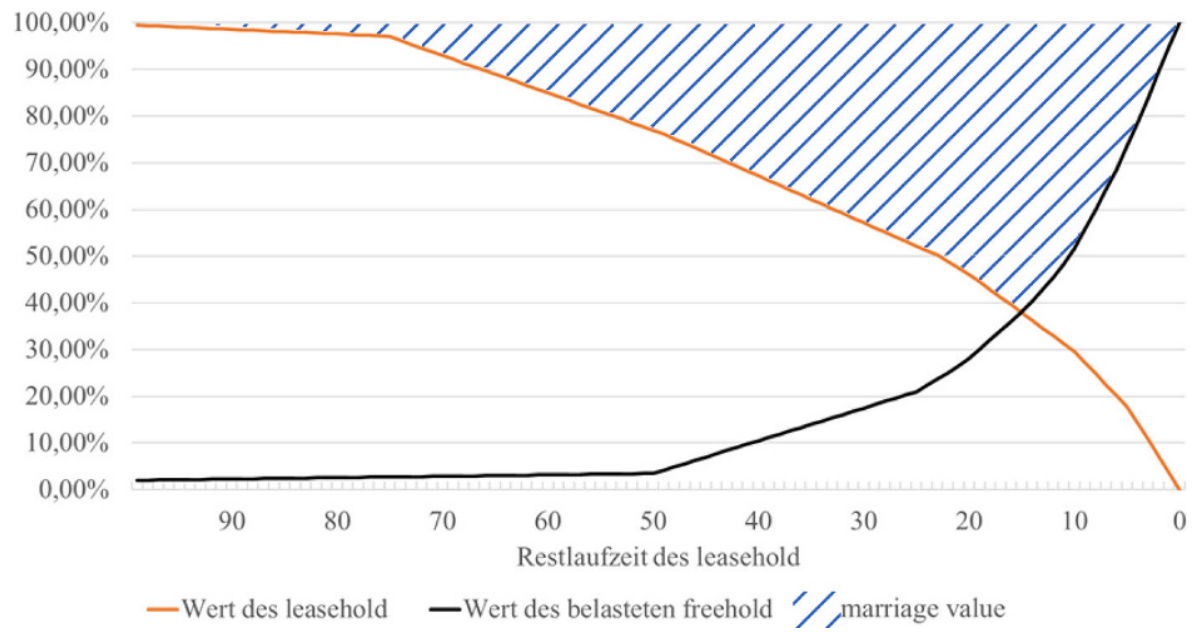

Abb. 8 Marriage Value Maximierung. (Eigene Darstellung in Anlehnung an Eisenhauer 1997, S. 121)

\footnotetext{
74 Vgl. Baum et al. (2018, S. 194).

75 Ein Erwerb des Erbbaurechts aus einem Zwangsversteigerungsfall heraus, kann die erwartete Rendite des Objekts zusätzlich erhöhen.
} 


\subsection{Wertermittlung nach dem Konzept des Marriage Value}

Mit dem Konzept des Marriage Value lässt sich die Verkehrswertermittlung umsetzen. Gemäß Eisenhauer (1997) besteht lediglich die Möglichkeit für einen positiven Marriage Value. Es sind allerdings je nach Marktbedingungen auch Konstellationen denkbar, in welchen ein negativer Marriage Value möglich ist. Dazu muss die Summe aus Wert des Leaseholds und Wert des belasteten Freeholds den Wert des unbelasteten Freeholds übersteigen. Das folgende Beispiel verdeutlicht die Zusammenhänge: ${ }^{76}$

Der Leasehold-Eigentümer eines im 19. Jahrhundert gebauten Londoner Wohnhauses möchte den Freehold erwerben. Der Leasehold hat eine 99-jährige Laufzeit und läuft seit dem 01.01.1927. Der Stichtag der Wertermittlung ist der 01.01.2018. Die weiteren Daten des Gebäudes sind folgende (Tab. 7).

Anhand der Daten vergleichbarer Transaktionen unbelasteter Grundstücke in der Nachbarschaft wurde ein aktueller Marktwert des Grundstücks als unbelasteter Freehold von 850.000 Pfund ermittelt. Abgeleitet aus einem Graph of Relativity für London ergibt die neunjährige Restlaufzeit einen Wert des Leasehold von $27 \%$ des Werts des unbelasteten Freehold, gerundet 230.000 Pfund. Der Wert des belasteten Freehold ergibt sich aus dem mit der Cap-Rate abgezinsten Zahlungsstrom der Ground Rent ${ }^{77}$, sowie dem anhand der Deferment Rate von 4,75\% ermittelten Wert des Freehold nach Ablauf des Lease:

Wert des belasteten Freehold $=£ 33+\frac{£ 850.000}{1,0475^{9}} \approx £ 559.832$

Der Marriage Value ergibt sich in diesem Beispiel wie folgt:

$$
\text { Marriage Value }=£ 850.000-(£ 230.000+£ 559.832)=\underline{\underline{£ 60.168}} .
$$

Anhand dieses Beispiels wird erkennbar, dass der Wert des belasteten Freehold maßgeblich durch die Deferment Rate bestimmt wird. Diese basiert auf einer regressionsbasierten Schätzung, welche sich an inflationsindexierten Staatsanleihen, Risikoprämie sowie langfristiger Wachstumsrate des Grundstückswertes orientiert. Eine weitere Stellschraube, um den Wert, der sich durch das Verfahren des Marriage Value ergibt, zu beeinflussen, stellen die Graphs of Relativity dar. In der Praxis sind verschiedene Graphen im Einsatz, die sich je nach veröffentlichender Institution und betrachtetem Markt unterscheiden. Des Weiteren stellt sich die Datenlage für

Tab. 7 Beispiel Marriage Value

\begin{tabular}{ll}
\hline Restlaufzeit des Leasehold & 9 Jahre \\
Ground Rent & $£ 5$ p. a. \\
Cap Rate & $7 \%$ \\
Deferment Rate & $4,75 \%$ \\
\hline
\end{tabular}

$76 \mathrm{Vgl}$. Shapiro et al. (2013).

$7733 \approx 5 . * \frac{1,07^{9}-1}{1,07^{9} *(1,07-1)}$ 
kurzfristige Restlaufzeiten bis 10 Jahre als schwierig dar. ${ }^{78}$ Zusätzlich ist die Existenz des Rechts, ein Leasehold Enfranchisement durchzuführen, der Grund für die Schwierigkeiten der Praxis bei der Ermittlung der Graphs of Relativity. ${ }^{79}$

Um den Marriage Value bestimmen zu können, ohne dass es in Erwartung seiner Existenz zu Verzerrungen kommt, wären Transaktionsdaten zu Leaseholds notwendig, für die kein Recht auf Enfranchisement besteht. Da aber heutzutage nahezu alle Leaseholds zu einem Enfranchisement berechtigt sind, existieren entsprechende Daten nicht in ausreichender Menge. Die in der Bewertungspraxis verwendeten Graphs of Relativity werden anhand unterschiedlicher Methoden ermittelt. Diese beinhalten neben historischen Daten von vor Inkrafttreten des Act 1993 auch Daten aus Gerichtsentscheidungen sowie Verhandlungsergebnisse oder Markteinschätzungen von Immobilienbewertern. ${ }^{80}$ Eine von der RICS eingesetzte Arbeitsgruppe zur Lösung dieser Schwierigkeiten konnte kein endgültiges Ergebnis erzielen. ${ }^{81}$

Ein negativer Marriage Value ist nach dieser Vorgehensweise technisch nicht möglich, da der Wert des Leaseholds und der Wert des belasteten Freeholds rechnerisch so voneinander abhängen, dass diese nicht die Summe des unbelasteten Freehold übersteigen können. Eine Änderung des einen Faktors bedingt eine gleichzeitig proportionale Änderung des anderen Faktors.

Der Vergleich der Wertermittlungsverfahren in Deutschland und Großbritannien zeigt auf, dass die jeweiligen Verfahren stets mit gewissen unsicherheitsbehafteten Faktoren einhergehen. Das Potenzial, einen Mehrwert zu generieren, hängt somit von besagten Stellgrößen ab. Im Fall der deutschen Wertermittlung sind dies Vergleichswert- und Marktanpassungsfaktoren. Für Großbritannien zeigten sich vor allem die Graphs of Relativity sowie die Deferment Rate als wertbeeinflussende Variablen.

\section{Erbbaurecht - Ein sinnvolles Nischenprodukt?}

Der Erwerb eines Erbbaurechtsgrundstücks ermöglicht eine direkte Investition in Grund und Boden bei gleichzeitiger Vermeidung unmittelbarer Vermietungs-, Instandhaltungs- und Modernisierungsrisiken. Inflationsrisiken werden durch Indexierung der Erbbauzinsen üblicherweise ebenfalls vermieden. ${ }^{82}$ Der Erbbauzins ist somit grundsätzlich ein stabiler, langjähriger Cashflow mit hoher Planungssicherheit, der allerdings von der Bonität des Erbbaurechtsnehmers abhängig ist. Bei ausbleibenden Zahlungen kann jedoch auf die Bebauung des Erbbaurechtsgrundstücks

\footnotetext{
78 Vgl. RICS (2009, S. 8).

79 Vgl. Grover (2014, S. 647-651).

80 Vgl. RICS (2009, S. 7).

81 Vgl. RICS (2009, S. 4).

82 Allerdings ist die Kappungsgrenze gemäß §9a ErbbauRG zu beachten. Hierbei darf eine Erhöhung des Erbbauzinses nicht über den Mittelwert aus der Entwicklung von Bruttolöhnen und Verbraucherpreisindex hinausgehen. Handelt es sich beispielsweise um einen Vertrag mit Inflationsklausel bei aktueller Inflation von $2 \%$, während im selben Zeitraum durchschnittlich die Bruttolöhne und Lebenshaltungskosten um $1 \%$ gestiegen sind, darf der Erbbauzins nur um 1,5\% angepasst werden. Zusätzlich muss bei Erbbaurechten zu Wohnzwecken die gesetzliche Höchstanpassung geprüft werden. Der gesetzlich zulässige Anpassungsbetrag stellt die Obergrenze der möglichen Kappung dar.
} 
zurückgegriffen werden. Der Erbbauzins ist zudem durch erstrangige Grundbucheintragung in den Aufbauten besichert. ${ }^{83}$

Die Investition in ein Grundstück mit bestehendem Erbbaurecht kann in einem Multi-Asset-Portfolio dazu beitragen, ein ausgewogenes Rendite-Risiko-Verhältnis zu erzielen. Auch wenn dies grundsätzlich für jede zusätzliche Anlageklasse gilt, weisen Erbbaurechtsgrundstücke besonders niedrige Volatilitäten bei vergleichsweise hoher Rendite aus.

Allerdings birgt das Investmentvehikel Erbbaurecht auch gewisse Risiken und zeigt bestimmte Grenzen auf.

Risikoadäquate und marktgerechte Verzinsung Aufgrund der geringen Markttransparenz sind Aussagen für Deutschland nur bedingt möglich. Die wenigen empirischen Befunde deuten jedoch darauf hin, dass Erbbauzinsen entsprechend dem allgemeinen Kapitalmarktumfeld ebenfalls gefallen sind. Die mögliche Inflationsindexierung des Erbbauzinssatzes könnte zur Folge haben, dass gegenüber Staatsanleihen ein Renditeabschlag droht. Bonitätsrisiken und Illiquidität begründen hingegen einen Zuschlag. Der Verlauf der Erbbauzinsen in NRW gegenüber dem Zinsverlauf (siehe Abb. 2) lässt jedoch eher vermuten, dass die Bepreisung von Erbbaurechten weitgehend vom Kapitalmarkt entkoppelt ist.

Erbbaurechte werden von Kommunen in Deutschland in der Regel zu einem Erbbauzins von 3-4\% des Bodenwertes vergeben. Eine Anpassung an veränderte Marktbedingungen, insbesondere an die Entwicklung der langfristigen Kapitalmarktzinsen, ist nicht zu erkennen. Auch ist nach unserem Kenntnisstand eine zwischenzeitliche Anpassung des Bodenwertes, die zumindest bei gewerblich genutzten Grundstücken möglich ist, derzeit nicht üblich. Aufgrund des niedrigen Zinsumfelds bewirkt eine nicht marktkonforme Festsetzung der Erbbauzinsen eine sinkende Attraktivität der Erbbaurechte für Erbbaurechtsnehmer bzw. für steigende Attraktivität für Erbbaurechtsgeber. Nach Löhr (2017) setzen Stiftungen und Kirchen die Erbbauzinsen zumindest marktnäher als Kommunen fest. Nur institutionell-kommerzielle Ausgeber von Erbbaurechten sollen sich marktkonform verhalten. Der Markt für Erbbaurechte ist insoweit vom Kapitalmarkt entkoppelt.

Fehlende Partizipation an Bodenwertsteigerung Aus Investorensicht ist eines der Hauptargumente, welches für Erbbaurechtsgrundstücke spricht, die konstante und risikoarme Verzinsung. Sofern aber die Inflationsrate deutlich niedriger als die Grundstückspreisentwicklung ist, kann dies zum wirtschaftlichen Nachteil für den Grundstückseigentümer werden. Zwar ist eine Indexierung des Erbbauzinses mit der Inflationsrate möglich, eine zwischenzeitlich eingetretene Wertsteigerung des Grundstücks bleibt jedoch in der Regel unberücksichtigt; bei Nutzung zu Wohnzwecken sogar aufgrund zwingender Rechtslage.

Von der zwischenzeitlichen Steigerung des Bodenwertes profitiert daher zunächst nur der Erbbaurechtsnehmer, sofern sich diese auch in höheren Erträgen niederschla-

${ }^{83}$ Erbbauzinsen sind bei vermieteten Objekten abzugsfähiger Zinsaufwand bei der Körperschafts- und Einkommensteuer. Sie fallen nicht unter die steuerliche Schuldzinsengrenze (,Zinsschranke“) für Unternehmen. 
gen. So kommt es vor, dass der Erbbaurechtsnehmer einen vergleichsweise geringen Erbbauzins für das von ihm genutzte Grundstück zahlt. Der Erbbaurechtsgeber muss hingegen bis zum Laufzeitende warten, um von der Bodenwertsteigerung zu profitieren. Aufgrund der sehr langen Laufzeiten kann die Bodenwertsteigerung auch nicht durch den Verkauf des Grundstücks realisiert werden. Durch diese Konstellation kann u.U. auch der Erwerb von Erbbaurechten eine attraktive Alternative zum Volleigentum darstellen.

Unter den Aspekt der Partizipation an Wertsteigerungen erscheint eine Investition in gewerblich genutzte Erbbaurechtsgrundstücke aufgrund der größeren Vertragsfreiheit sowohl in Deutschland als auch in Großbritannien deutlich attraktiver.

Kleiner Markt für Erbbaurechtsgrundstïcke und Erbbaurechte Der Markt mit Erbbaurechtsgrundstücken und Erbbaurechten hat (vermutlich) nur einen geringen Anteil am Gesamtmarkt. Zudem sind diese Marktsegmente im Vergleich zu Volleigentum deutlich intransparenter. Hieraus kann sich eine zusätzliche Illiquidität von Erbbaurechten und Erbbaurechtsgrundstücken ergeben. Bei effizienten Märkten würde dies zusätzliche Illiquiditätsprämien rechtfertigen. Erbbaurechte eignen sich aufgrund ihrer Illiquidität somit eher für Institutionen mit geringeren Anforderungen an die Liquidität der Assets, beispielsweise aufgrund sehr langer Planungshorizonte.

Der Nutzen für Investoren hängt insbesondere in Deutschland stark von der konkreten Vertragsgestaltung ab. Es sind dingliche und schuldrechtliche Vereinbarungen, Vorkaufsrechte, Berechnungsverfahren sowie etwaige andere Verpflichtungen zu überprüfen. ${ }^{84}$ Aufgrund der Komplexität übersteigt für Privatpersonen der Aufwand häufig den Nutzen. Hingegen sind institutionelle Investoren ein prädestinierter Anlegerkreis wegen des Potenzials zu höherem Know-How und den finanziellen und zeitlichen Gestaltungsspielräumen.

Auch der britische Markt für Erbbaurechte ist deutlich besser etabliert als der deutsche. Daher erscheint der britische Markt aufgrund der höheren Liquidität besser geeignet für institutionelle Investoren. Auch das Volumina der auf Erbbaurecht spezialisierten Immobilienfonds in Großbritannien zeigt das Marktpotenzial auf, das deutsche Fonds bisher nur in geringem Maße nutzen konnten. Für volumenstarke innovative Investoren ergeben sich aber in Deutschland gerade aufgrund der Marktenge und der Intransparenz besondere Chancen. Diese können zum einen mit wenigen Transaktionen bzw. Aufspaltungen den Markt wesentlich beeinflussen. Sofern es zum anderen gleichzeitig gelingt, für Erbbaurecht und Erbbaurechtgrundstücke Investoren mit entsprechend kompatiblen Investitionsprofilen zu identifizieren, können durch Aufspaltungen zusätzliche Wertsteigerungen generiert werden.

\footnotetext{
${ }^{84}$ Durch die aktuelle Gesetzeslage ist in Deutschland - im Gegensatz zu Großbritannien - noch kein Standard etabliert. Als wichtige Aspekte sind in Deutschland die Zusicherung bzw. Vorkaufsrechts nach \$2 Nr. 7 ErbbauRG und die Ermittlung der Entschädigung (analog zum Enfranchisement) vertraglich zu fixieren.
} 
Interessenkonflikt S. Sebastian, D. Wagner und C. Fritz geben an, dass kein Interessenkonflikt besteht.

Open Access Dieser Artikel wird unter der Creative Commons Namensnennung 4.0 International Lizenz veröffentlicht, welche die Nutzung, Vervielfältigung, Bearbeitung, Verbreitung und Wiedergabe in jeglichem Medium und Format erlaubt, sofern Sie den/die ursprünglichen Autor(en) und die Quelle ordnungsgemäß nennen, einen Link zur Creative Commons Lizenz beifügen und angeben, ob Änderungen vorgenommen wurden.

Die in diesem Artikel enthaltenen Bilder und sonstiges Drittmaterial unterliegen ebenfalls der genannten Creative Commons Lizenz, sofern sich aus der Abbildungslegende nichts anderes ergibt. Sofern das betreffende Material nicht unter der genannten Creative Commons Lizenz steht und die betreffende Handlung nicht nach gesetzlichen Vorschriften erlaubt ist, ist für die oben aufgeführten Weiterverwendungen des Materials die Einwilligung des jeweiligen Rechteinhabers einzuholen.

Weitere Details zur Lizenz entnehmen Sie bitte der Lizenzinformation auf http://creativecommons.org/ licenses/by/4.0/deed.de.

\section{Literatur}

Aholt H, Hartl B (2016) Deloitte, Ermittlung marktgerechter Erbbauzinsen, Vortrag beim 3. Erbbaurechtskongress des Deutschen Erbbaurechtsverbandes, Hannover

Alternative Real Capital (2018) Index linked income fund

Astberrys (2009) What price do you put in marriage? The concept of "marriage value" in a lease of less than 80 years

Baum A, Mackmin D, Nunnington N (2018) The income approach to property valuation. Routledge, Abingdon

Böttcher R (2017) Praktische Fragen des Erbbaurechts. RWS Verlag Kommunikationsforum GmbH, Köln

Bundesanstalt für Finanzdienstleistungen (BaFin) (2009) Beleihungswertermittlung bei Erbbaurechten

Bundesministerium für Umwelt, Naturschutz, Bau \& Reaktorsicherheit (2006) Wertermittlungsrichtlinien - Anlagen, S 12-22

Bürgerschaftsfraktion Hamburg SPD (2019) Neuausrichtung der Bodenpolitik

Christoph C (2017) Perspektive Europa. Rote Seiten: Erbbaurecht - Neue Perspektiven auf einen Klassiker. Issue 03

Collinson, P., 2017. Leasehold in England and Wales is last redoubt of a colonial relic. The Guardian, Issue 26. Juli 2017.

Department for Communities and Local Government (2017a) Estimating the number of leasehold dwellings in England

Department for Communities and Local Government (2017b) Tackling unfair practices in the leasehold market. The National Archives, London

Deutscher Erbbaurechtsverband e. V. (2018) Studie zum Erbbaurecht - Befragung von Erbbaurechtsgebern. Analyse \& Konzepte, Hamburg

Direct Line Insurance Group (2016) Property pain: service charges increasing rapidly. Direct Line Group, Leeds

Dixon M (2016) Modern land law Bd. 10

DNotI-Report (2001), Band 22, S. 177-184, Informationsdienst des Deutschen Notarinstituts, Würzburg

Driscoll J (2016) Whatever happend to commonhold?

Eisenhauer M (1997) Moderne Entwicklungen im Englischen Grundstücksrecht. Mohr Siebeck, Tübingen

Ernst \& Young (2017) Ground rents: an opportunity for institutional investors to diversify exposure. EYGM Limited, Eschborn/Frankfurt am Main

GlobalCapital (2006) Vivacon German ground rent securitisation is market first

Grizwotz H (2017) Erman BGB - Das renommierte Standardwerk zum BGB. Dr. Otto Schmidt, Köln

Grover R (2014) Leasehold enfranchisement and graphs of relativity. J Prop Invest Finance 32(6):642-652

Hansen K (2006) Pressemitteilung HVB

Hüren D (2015) Eigenes Heim auf fremdem Boden - Fragen zum Erbbaurecht - Frankfurter Neue

Hustedt V, Ingenstau J (2014) ErbbauRG Gesetz über das Erbbaurecht. Carl Heymanns, Köln

Ingold M (2017) Bewertung von Erbbaurechten in der Niedrigzinsphase. Der Immobilienbewerter/ Zeitschrift für die Bewertungspraxis 2017(2):3-11 
Institute and Faculty of Actuaries (2015) Non-traditional investments-key considerations for insurers. Non-traditional investments Working Party, London

Keilhauer O (2017) Einsatz von Erbbaurechtsmodellen zur Optimierung des Anlageerfolgs. Rote Seiten: Erbbaurecht - Neue Perspektiven auf einen Klassiker

Kleiber W (2017) Verkehrswertermittlung von Grundstïcken. Kommentar und Handbuch, 8. Aufl. Bundesanzeiger-Verlag, Köln

Landeshauptstadt München (2017) Referat für Stadtplanung und Bauordnung, 2017. Wohnen in München VI 2017-2021. Wohnungspolitisches Handlungsprogramm

Lang LaSalle J (2019) Erbbaurechte in deutschen Metropolen

Licher T (2009) Das Erbbaurecht - Königsweg für Kommunen: Studie der Initiative Erbbaurecht

Löhr D (2017) Zur Ermittlung eines marktgerechten Erbbauzinses - ein Praktikermodell. Z Immobilienökon 3:1-19

Long Harbour Ltd (2018) Long harbour ground rent fund

McNeill S, Williamson D (2017) Out-Law.com: securitisation dispute should remind trustees of their duty of impartiality, says expert

Meusel S (2017) Beitrag zum Thema Erbbaurecht in der Zeitrschift Stiftung \& Sponsoring Rote Seiten. In: DSZ - Deutsches Stiftungszentrum GmbH (Hrsg) Ausgewählte Fallen im Erbbaurechtsvertrag Das Nachbarerbbaurecht und andere rechtliche Fallstricke des Erbbaurechtsvertrages. Erich Schmidt, Berlin

My Home Move Ltd (2016) London and North-West emerge as England and Wales' leasehold hotspots

Nagel M (2017) Erbbaurecht als Instrument der Vermögensanlage für Stiftungen. Rote Seiten: Erbbaurecht - Neue Perspektiven auf einen Klassiker, Bd. 3

Oberer Gutachterausschuss für Grundstückswerte im Land Nordrhein-Westfalen (2017) Grundstücksmarktbericht 2017

von Oefele H, Winkler K (2012) Handbuch des Erbbaurechts. C. H. Beck, München

von Oefele H, Winkler K, Schlögel J (2016) Handbuch des Erbbaurechts. C. H. Beck, München

Private Banking Magazin (Hrsg) (2012) Inflationssicherheit mit Erbbau-Grundstücken. Franklin Templeton Investments, Hamburg

RICS (2009) Leasehold reform: graphs of relativity

Runder Tisch Liegenschaftspolitik (2017) Erbbaurechte in Berlin - Dokumentation der Expertenwerkstatt. Stiftung trias, Berlin

Savills Research (2014) Spotlight - alternative residential investments. Savills World Research - UK Residential, London

Schmenger W (2006) Aktuelle Rechtsfragen beim Erbbaurecht. Z Notar Baden Württemb 4:73-96

Shapiro E, Mackmin D, Sams G (2013) Modern methods of valuation. Routledge, Abingdon

TEGOVA - The European Group of Valuers' Associations (2016) Europäische Bewertungsstandards

The Leasehold Reform Regulations (1993) Collective enfranchisement and lease renewal. The Leasehold Reform Regulations, London

Waldner W, Kopp B (2015) Handbuch Immobilienrecht in Europa. C.F. Müller, Heidelberg

Wilson W, Barton C (2018) Leasehold and commonhold reform

Wilson W, Barton C (2019) Leasehold and commonhold reform. House of Commons Library, 12 April, Issue Number 8047, London 